\title{
$\delta$-exceedance records and random adaptive walks
}

\author{
Su-Chan Park ${ }^{1}$ and Joachim Krug ${ }^{2}$ \\ ${ }^{1}$ Department of Physics, The Catholic University of Korea, Bucheon 14662, Republic \\ of Korea \\ ${ }^{2}$ Institut für Theoretische Physik, Universität zu Köln, Köln 50937, Germany
}

10 October 2018

\begin{abstract}
We study a modified record process where the $k^{\prime}$ th record in a series of independent and identically distributed random variables is defined recursively through the condition $Y_{k}>Y_{k-1}-\delta_{k-1}$ with a deterministic sequence $\delta_{k}>0$ called the handicap. For constant $\delta_{k} \equiv \delta$ and exponentially distributed random variables it has been shown in previous work that the process displays a phase transition as a function of $\delta$ between a normal phase where the mean record value increases indefinitely and a stationary phase where the mean record value remains bounded and a finite fraction of all entries are records (Park et al 2015 Phys. Rev. E 91 042707). Here we explore the behavior for general probability distributions and decreasing and increasing sequences $\delta_{k}$, focusing in particular on the case when $\delta_{k}$ matches the typical spacing between subsequent records in the underlying simple record process without handicap. We find that a continuous phase transition occurs only in the exponential case, but a novel kind of first order transition emerges when $\delta_{k}$ is increasing. The problem is partly motivated by the dynamics of evolutionary adaptation in biological fitness landscapes, where $\delta_{k}$ corresponds to the change of the deterministic fitness component after $k$ mutational steps. The results for the record process are used to compute the mean number of steps that a population performs in such a landscape before being trapped at a local fitness maximum.
\end{abstract}

Keywords: record process, extreme value theory, evolutionary dynamics, epistasis, fitness landscape

Submitted to: J. Phys. A: Math. Theor.

\section{Introduction}

\subsection{Record processes}

The mathematical theory of records is concerned with the statistics of extremes in a time series of random observations. In the standard setting, an entry $X_{n}$ in the series is a (upper) record if it exceeds all previous entries, i.e. if $X_{n}>\max \left\{X_{1}, X_{2}, . ., X_{n-1}\right\}$. When the $X_{n}$ 's are independent and identically distributed (i.i.d.) random variables, 
the properties of the sequence of record times and record values have been studied in great detail and are now well understood [1, 2, 3]. If the common distribution function $F$ of $X_{n}$ is continuous, the statistics of record times is completely universal regardless of the choice of $F$. In particular, the number of records up to time $n$ is asymptotically equal to $\ln (n)[1,2,3]$.

In many applications of record theory the observations are subject to uncertainty and the definition of record occurrence needs to be modified [4]. Two basic situations are conceivable. On the one hand, to make sure that spurious records caused by measurement error are not counted, one demands that the new record should exceed the old one at least by an amount $\delta>0$. On the other hand, to avoid missing any potential events of interest, one relaxes the record condition and includes observations in the record sequence that are smaller than the previous record by at most $\delta$. Both situations have been invoked to motivate the study of $\delta$-records defined by the condition [5, 6]

$$
X_{n}>\max \left\{X_{1}, X_{2}, \ldots, X_{n-1}\right\}+\delta .
$$

Specifically, for $\delta<0$ the events satisfying (1) are referred to as near-records [7]. An immediate consequence of introducing the parameter $\delta$ is that the strong universality of the statistics of record times is lost and replaced by an explicit dependence on the tail properties of the underlying distribution $F$, similar to other modified record processes involving discreteness [8, 9], rounding effects [10] or trends [11, 12].

Importantly, according to (1) the threshold that the new record has to exceed is defined in terms of the "true" record sequence, the maximum process $M_{n} \equiv$ $\max \left\{X_{1}, X_{2}, . ., X_{n}\right\}$, which (under the conditions of measurement uncertainty described above) may not even be observable. A more faithful representation of the measurement error scenario, in which the next record occurs conditional on the previous observed record, was introduced twenty years ago by Balakrishnan et al. under the name of $\delta$ exceedance records [13. They considered the case $\delta>0$ and derived several results for the case when the underlying distribution $F$ is of exponential or Gumbel form. Mainly for reasons of mathematical tractability [4], subsequent work has however focused on the problem of $\delta$-records defined by (1).

A qualitative difference between $\delta$-records and $\delta$-exceedance records arises in the case when $\delta<0$; see figure 1. Because $\delta$-records are coupled to the maximum process $M_{n}$, the exceedance threshold for a new record defined in (1) increases monotonically in time. Although the records themselves do not necessarily grow monotonically, the growing threshold ensures that the record values increase on average and are pushed into the tail of $F$. By contrast, in the $\delta$-exceedance record process (to be defined in precise mathematical terms in section 2) the threshold may decrease when $\delta<0$. This entails the possibility that a finite fraction of entries in the time series are counted as records and the expected record value remains asymptotically bounded even for an unbounded distribution. We will refer to this behavior as the stationary phase of the record process.

In a recent publication we showed that this scenario is indeed realized and leads to 


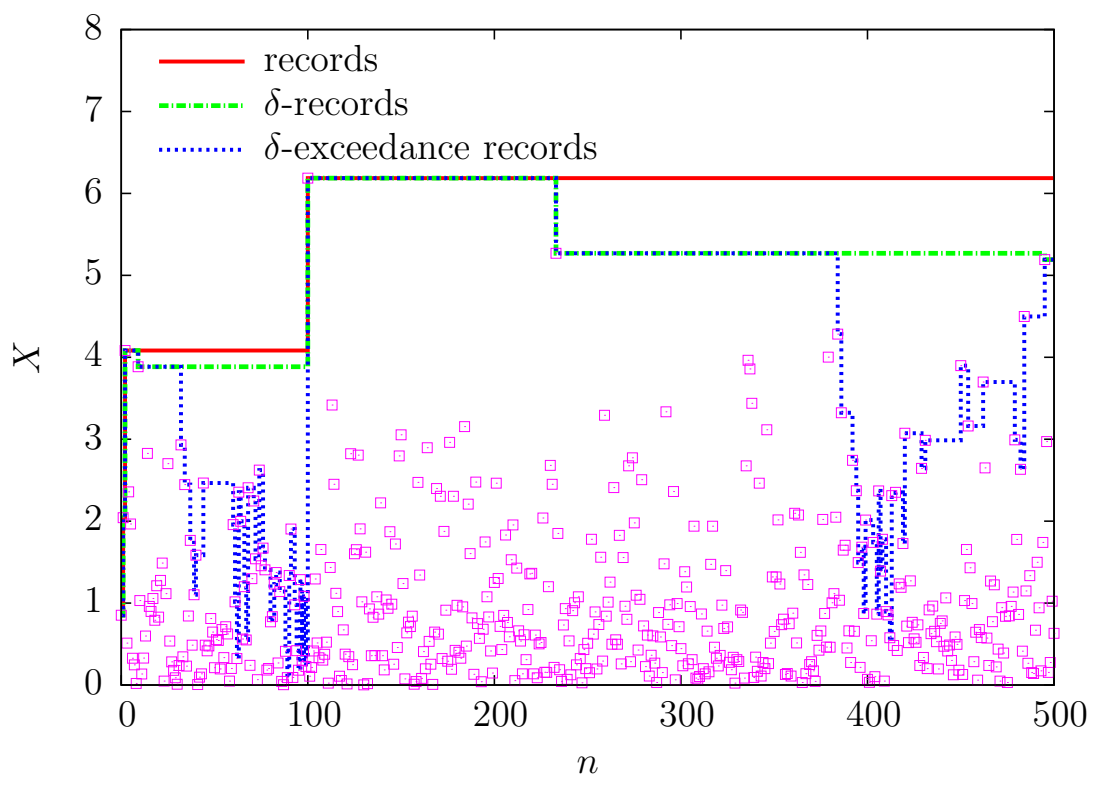

Figure 1. Comparison of sample paths of the standard record process (red solid line), $\delta$-records (green dot-dashed line), and $\delta$-exceedance records (blue dotted line) for the same realization of the background process. Random variables of the background process drawn from an exponential distribution with unit mean are shown as symbols. In this example, $\delta$ is set to -1 , which corresponds to the critical point of the $\delta$ exceedance process. Note the cascades of decreasing record values which signal the incipient stationary phase that emerges when $\delta<-1$.

a novel kind of phase transition as a function of $\delta$ when the underlying distribution has an exponential tail [14]. Our results were obtained in the context of adaptive walks in biological fitness landscapes, which we explain next.

\subsection{Adaptive walks}

Adaptive walks are simple evolutionary dynamics defined on a space of genotypes [15, 16, 17, 18, 19, 20, 14]. In the most common setting genotypes are encoded by binary sequences of length $L$, where each letter denotes the presence of one of two alleles (say, 0 or 1) at a given genetic locus or nucleotide position. Genotypes are assigned real fitness values which quantify the reproductive potential of the corresponding individuals. In one step of the walk, neighboring genotypes of higher fitness are sampled and one of them is chosen as the next position of the walker. Here two genotypes are defined to be neighbors if they differ by a single point mutation, that is, at one site of the sequence. If no fitter neighbors exist the population has reached a local fitness maximum and the walk stops. Quantities of interest in the theory of adaptive walks are the number of steps required to reach a local maximum and the fitness value that has been reached at this point.

In the present work we focus on the conceptually simplest case of the random adaptive walk (RAW) where the next genotype along the walk is chosen at random among the neighbors of higher fitness. Let us assume that the fitness values of different 
genotypes are i.i.d. random variables and consider the limit $L \rightarrow \infty$. In this limit there are no local fitness maxima and the walk progresses indefinitely. At each step the walk moves to a new fitness value which is a random draw from the underlying fitness distribution conditioned on being larger than the previous value. Thus, the fitness values encountered along the walk form a series of records.

To estimate the number of steps that the RAW takes when $L$ is finite, we note that the walker will stop when the fitness value that it has reached exceeds the maximum among $L$ i.i.d. random variables. In the record picture this means that the time at which the RAW stops is of order $L$. At this point the number of records, that is, the number of RAW steps, is of order $\ln (L)$, in agreement with the detailed analysis [16, 17]. In fact, the sequence length $L$ and the time $n$ in the i.i.d. record problem are exactly equivalent in a variant of the RAW model where the set of available neighboring genotypes is kept fixed throughout the walk [18, 19].

In [14 we considered RAW's in a setting where i.i.d. random fitness values are added to a deterministic fitness profile. The walker is assumed to start in a state of low fitness and every step brings it closer to a high fitness peak. Denoting the genotype that has been reached after $k$ steps by $\mathcal{C}_{k}$ and the corresponding fitness by $W\left(\mathcal{C}_{k}\right)$, the model is defined by

$$
W\left(\mathcal{C}_{k}\right)=g_{k}+\eta_{\mathcal{C}_{k}},
$$

where $g_{k}$ is a deterministic, monotonically increasing function of $k$ and the $\eta_{\mathcal{C}}$ 's are i.i.d. random variables associated with genotypes. The condition for a genotype $\mathcal{C}^{\prime}$ to be a possible target for the next step of the RAW then reads $W\left(\mathcal{C}^{\prime}\right)=g_{k+1}+\eta_{\mathcal{C}^{\prime}}>W\left(\mathcal{C}_{k}\right)$ or, in terms of the i.i.d. random variables,

$$
\eta_{\mathcal{C}^{\prime}}>-\left(g_{k+1}-g_{k}\right)+\eta_{\mathcal{C}_{k}}
$$

For the special case of a linear fitness gradient $g_{k}=c k$, also known as the Rough Mount Fuji model [14, 21], the $L \rightarrow \infty$ RAW is thus seen to be equivalent to the $\delta$-exceedance record problem with $\delta=-c$.

\subsection{Goal and outline of the paper}

It was shown in [14] that the phase transition in the $\delta$-exceedance record process with $\delta<0$ occurs only when the common distribution of the i.i.d. random variables has an exponential tail. The special role of the exponential distribution reflects the wellknown fact that record values from exponentially-tailed distributions are asymptotically equally spaced [2]. Correspondingly, phase-transition like phenomena can be expected for other tail shapes if the constant $\delta<0$ is replaced by an offset that varies with the number of records, and some preliminary results along these lines were reported in [14].

The purpose of this paper is to provide a comprehensive analysis of the generalized $\delta$-exceedance record problem with a monotonically varying, negative offset. For notational convenience, we will denote the offset associated with the $k$ 'th record by $-\delta_{k}\left(\delta_{k}>0\right)$. Moreover, because the offset facilitates the establishment of new records, 
$\delta_{k}$ will be referred to as the 'handicap'. Results covering all three extreme value classes of random variables and decreasing as well as increasing handicap will be presented.

In the context of adaptive walks, the variation of $\delta_{k}=g_{k+1}-g_{k}$ with $k$ implies that the deterministic effect of a mutation depends on where it occurs along the evolutionary trajectory, a phenomenon known as epistasis [22]. In particular, a pattern of diminishing returns epistasis where mutational effect sizes decrease with the number of adaptive steps is commonly observed in evolution experiments with microbial populations [23, 24, 25, 26, 27].

In the next section we define the generalized $\delta$-exceedance record problem considered in this work, and we specify the probability distributions of the underlying random variables that will be used. The case of decreasing handicaps is examined in section 3 and the case of increasing handicaps in section 4 . In section 5 we use the results for the record process to obtain estimates for the length of adaptive walks. Finally, in section 6 we summarize our findings and provide some conclusions in the contexts of record statistics as well as evolutionary dynamics.

\section{Statement of the problem}

Consider a sequence $\left\{X_{i}, i \geq 0\right\}$ of i.i.d. random variables with a common distribution function $F$ and probability density $f$. From this sequence, we construct recursively the generalized $\delta$-exceedance record process $\left\{Y_{k}, k \geq 0\right\}$ and the corresponding recordoccurrence-time process, $\left\{n_{k}, k \geq 0\right\}$, as follows. We first define $Y_{0}=X_{0}$ and $n_{0}=0$. Suppose that $Y_{l}$ and $n_{l}$ up to $l=k-1(k=1,2, \ldots$,$) have been determined. We then$ define $n_{k}$ as

$$
n_{k}=\min \left\{i \mid X_{i}>Y_{k-1}-\delta_{k-1}, i>n_{k-1}\right\},
$$

where $\delta_{k}$ is a deterministic $k$-dependent sequence that will be called the handicap. Once $n_{k}$ is determined, we set $Y_{k}=X_{n_{k}}$. For later purposes, $\left\{X_{i}, i \geq 0\right\}$ will be referred to as the background process.

In the following, we set

$$
\delta_{k}=c(k+1)^{b-1}
$$

with $b>0$. If $b>1(b<1)$, the handicap $\delta_{k}$ increases (decreases) with $k$. An epistatic fitness landscape model of the form (5) has been considered in [28].

The following three kinds of distributions with parameters $a, r, \alpha, \nu, \mu>0$ will be considered for the background process:

$$
\begin{aligned}
& F_{g}(x)=1-\exp \left(-x^{\alpha} / a\right), \\
& F_{w}(x)=1-(1-x / r)^{1 / \nu}, \\
& F_{f}(x)=1-(1+x / a)^{-\mu} .
\end{aligned}
$$

The subscripts $g, w, f$ refer to the Gumbel, Weibull, and Fréchet classes of extreme value theory, respectively [29]. The corresponding densities $f_{g}$ and $f_{f}$ have semi-infinite support $x>0$ and the support of $f_{w}$ is the interval $0<x<r$. 
As in Ref. [14], the mean value $z_{l}$ of the l'th record will turn out to play an important role in understanding the record process. To derive a recursion relation for $z_{l}$ we consider the probability density of $Y_{l}$, which is denoted by $Q_{l}$. It is a straightforward generalization of the case with $b=1$ [14] to obtain the recursion relation

$$
Q_{l+1}(y)=f(y) \int_{-\infty}^{y+\delta_{l}} \frac{Q_{l}(x)}{1-F\left(x-\delta_{l}\right)} d x
$$

with $Q_{0}(y)=f(y)$. As a consequence, $z_{l}$ satisfies (see Appendix A for the derivation)

$$
z_{l+1}=\left\langle\frac{1}{h}\right\rangle_{l+1}+\int_{\delta_{l}}^{R}\left(x-\delta_{l}\right) Q_{l}(x) d x,
$$

where $h$ is the hazard function defined as

$$
h(y) \equiv \frac{f(y)}{1-F(y)}=-\frac{d}{d y} \ln [1-F(y)],
$$

$R$ is the supremum of the support, and $\langle\ldots\rangle_{l}$ stands for the average with respect to $Q_{l}$. The hazard functions corresponding to the distributions (6) are

$$
\begin{aligned}
& h_{g}(x)^{-1}=a x^{1-\alpha} / \alpha, \\
& h_{w}(x)^{-1}=\nu(r-x), \\
& h_{f}(x)^{-1}=(a+x) / \mu .
\end{aligned}
$$

Note that (8) remains valid for $R=\infty$ and hence can be used for the distributions $F_{g}$ and $F_{f}$. Moreover, it can be shown that the last term in (8) becomes $z_{l}-\delta_{l}$ when the support of $f$ is unbounded on both sides and the recursion reduces to that considered in [14] (see Appendix A). The analyses in the following sections will be largely based on (8).

Before embarking on the detailed investigation, it can be instructive to develop a heuristic picture based on the behavior of the mean record value for the standard case. It is plausible to expect that the handicaps $\delta_{k}$ will be relevant (irrelevant) to the record process if they are asymptotically larger (smaller) than the mean difference between subsequent record values in the standard setting.

Consider first the Gumbel-type distributions $F_{g}$, for which the mean value of the $l$ 'th (standard) record is asymptotically equal to $(a l)^{1 / \alpha}[2]$ and hence the difference between subsequent record values is proportional to $l^{\frac{1}{\alpha}-1}$. Comparing to (5) it follows that the handicap should be relevant (irrelevant) for $b>1 / \alpha(b<1 / \alpha)$. The value $b=1 / \alpha$ is thus of special interest as it is the only case where an extension of the phase transition scenario described in [14] can be expected to arise. For the Fréchet class distributions $F_{f}$ the mean (standard) record value grows exponentially with $l$, whereas it approaches the upper boundary of the support exponentially fast for the Weibull class distributions $F_{w}$ [2]. Correspondingly, one expects the record statistics to always be asymptotically modified (unmodified) by the handicaps for distributions in the Weibull (Fréchet) classes. We will see below that these expectations are largely confirmed by the detailed analysis, but in addition several unanticipated features emerge. 


\section{Decreasing handicap}

When $b=1$ and $R=\infty, z_{l}$ either increases indefinitely with $l$ or saturates to a finite value, depending on the tail behavior of $F$ [14]. Expecting a similar behavior, we focus on determining the conditions under which $z_{l}$ diverges with $l$ when $b<1$. To this end, we first assume that $z_{l}$ indeed diverges. Another crucial assumption is that $Q_{l}(x)$ is sharply peaked around $z_{l}$ when $l$ is sufficiently large. Because this amounts to neglecting fluctuations around $z_{l}$, it will be referred to as the mean-field approximation (MFA). Under these assumptions, we can approximate the integral in (8) as $z_{l}-\delta_{l}$ and, in turn, we arrive at the approximate equation

$$
z_{l+1}-z_{l} \approx \frac{1}{h\left(z_{l+1}\right)}-\delta_{l} .
$$

When $R$ is finite, the above assumptions are clearly not applicable and we will use a different approach.

\subsection{Gumbel class}

We first consider the distribution $F_{g}(x)$. Using the corresponding hazard function in (10), we get

$$
z_{l+1}-z_{l} \approx \frac{a}{\alpha} z_{l+1}^{1-\alpha}-c(l+1)^{b-1} .
$$

Assuming that $z_{l}$ is a slowly varying function of $l$ in the sense that $\left(z_{l+1}-z_{l}\right) / z_{l} \rightarrow 0$ as $l \rightarrow \infty$, we can rewrite the above equation as a differential equation

$$
\frac{d}{d l}\left(\frac{z^{\alpha}}{a}\right)=1-c \frac{\alpha}{a} z^{\alpha-1} l^{b-1},
$$

where $z$ is now meant to be a continuous function of $l$. Since we are interested in the asymptotic solution, we set $z^{\alpha} \approx a(A l)^{\gamma}$, which yields

$$
A^{\gamma} \gamma l^{\gamma-1} \doteq 1-A^{1-b} \frac{c \alpha}{a^{1 / \alpha}}(A l)^{\gamma(1-1 / \alpha)+b-1},
$$

where $\doteq$ means that the equality holds only for the leading behavior on both sides. Thus, the asymptotic behavior of $z_{l}$ can be consistently determined by comparing powers, $\gamma-1$ and $\gamma(1-1 / \alpha)+b-1$, in (14) with 0 .

If $\gamma(1-1 / \alpha)+b-1>0$, the right hand side (RHS) of (14) will eventually become negative while the left hand side (LHS) is positive for any $l$. Accordingly, any consistent solution requires $\gamma(1-1 / \alpha)+b-1 \leq 0$. Likewise, if $\gamma>1$, no consistent solution exists because the LHS increases to infinity while the RHS cannot. Hence, we can conclude that only solutions with $\gamma \leq 1$ and $\gamma(1-1 / \alpha)+b-1 \leq 0$ are possible.

Let us first consider what will happen if the solution is $\gamma=1$. With this assumption, we rewrite (14) as

$$
A \doteq 1-A^{1-b} \frac{c \alpha}{a^{1 / \alpha}}(A l)^{b-1 / \alpha} .
$$


If $b<1 / \alpha$, we get $A=1$, which corresponds to the behavior of the standard record values (that is, $c=0$ ). If $b=1 / \alpha, A$ is the solution of equation

$$
A^{b-1}-A^{b}=\frac{c}{a^{b} b} \text {. }
$$

Note that as long as $b<1$ a positive solution of (16) uniquely exists in the interval $0<A \leq 1$ for any $c \geq 0$. In the following, we will denote this solution by $A_{b}(c)$.

Now we investigate if a solution with $\gamma<1$ exists. Since the LHS of (14) approaches 0 in this case, the RHS should also approach zero. Thus, we require that $\gamma=(1-b) /(1-1 / \alpha)$ and we get

$$
A=\left(\frac{c \alpha}{a^{1 / \alpha}}\right)^{1 /(b-1)}
$$

by equating the right hand size of (14) to zero. Note that for $\gamma$ to be smaller than 1 , $b>1 / \alpha$ should be satisfied.

Actually, the LHS of (14) with $\gamma<1$ gives rise to a subleading correction. To see this, let us set $z^{\alpha}=a(A l)^{\gamma}\left(1+B l^{-\beta}\right)$, where $A$ and $\gamma$ are the solutions for $\gamma<1$ in the above and $\beta>0$. Then we get

$$
A^{\gamma} \gamma l^{\gamma-1} \doteq-\frac{\alpha-1}{\alpha} B l^{-\beta}
$$

and hence

$$
\frac{z_{l}^{\alpha}}{a} \approx(A l)^{\gamma}\left[1-\frac{\alpha \gamma}{\alpha-1} A^{\gamma} l^{\gamma-1}\right]
$$

Since we neglect the effect of fluctuation of $Q_{l}$, the subleading term in (19) should however not be taken seriously.

To sum up, (12) has solutions of diverging $z_{l}$ for any $\alpha$ as long as $b<1$. The leading behavior of $z_{l}$ is

$$
\frac{z_{l}^{\alpha}}{a}=(A l)^{\gamma}
$$

where

$$
A= \begin{cases}1, & \alpha<1 / b, \\ A_{b}(c), & \alpha=1 / b, \\ \left(c \alpha / a^{1 / \alpha}\right)^{1 /(b-1)}, & \alpha>1 / b,\end{cases}
$$

and

$$
\gamma= \begin{cases}1, & \alpha \leq 1 / b \\ (1-b) /(1-1 / \alpha), & \alpha>1 / b .\end{cases}
$$

These results confirm the heuristic considerations of section 2 , For $\alpha<1 / b$ the record values behave asymptotically as in the standard case, i.e. the handicaps are irrelevant, whereas for $\alpha>1 / b$ the behavior is modified qualitatively and the record values grow more slowly than the standard record process. On the 'critical line' $\alpha=1 / b$ the mean record value grows with the same power as in the standard case but with a reduced prefactor $A_{b}(c)$ which interpolates smoothly between the limits $A_{b}(c=0)=1$ and 


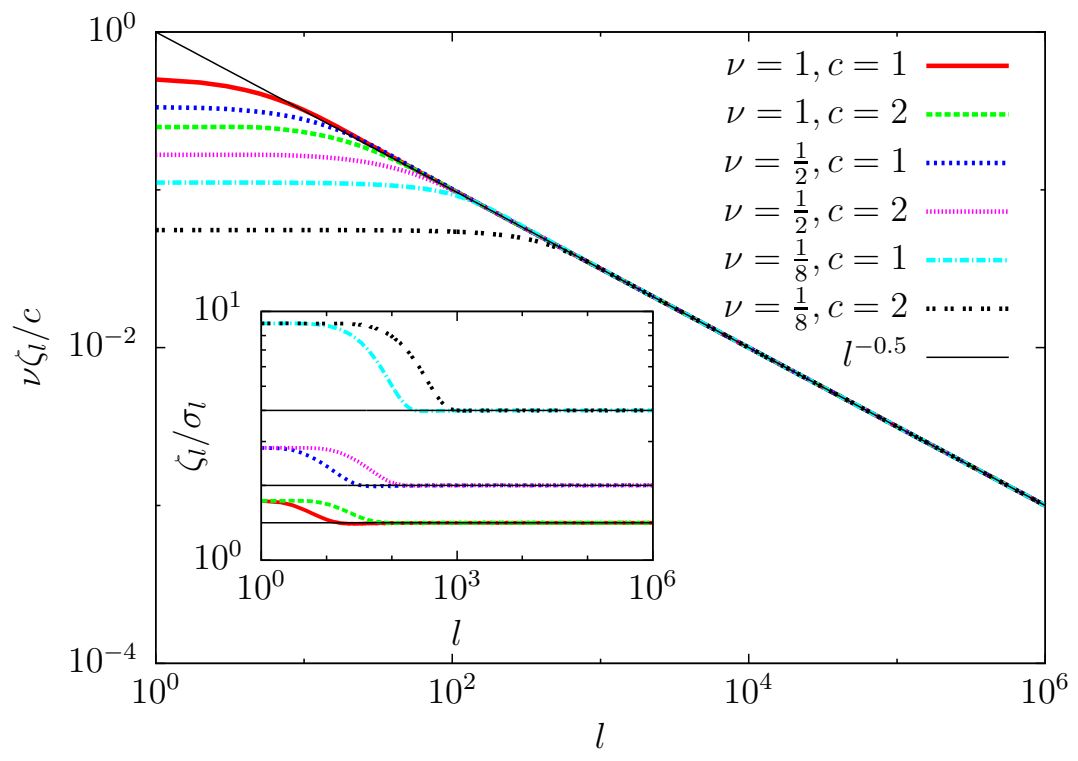

Figure 2. Illustration of the approach of the mean record value $z_{l}$ to the upper boundary $r$ of the support for Weibull-class distributions. The figure shows $\nu \zeta_{l} / c$ vs. $l$ for $b=\frac{1}{2}, \nu=1, \frac{1}{2}$, and $\frac{1}{8}$ with $c=1$ and 2 on a double logarithmic scale. Here $\zeta_{l}=r-z_{l}$. For comparison, we plot the predicted behavior $l^{-1 / 2}$. Inset: Double-logarithmic plots of $\zeta_{l} / \sigma_{l}$ against $l$ for various $\nu$. The horizontal lines show the predicted value $\sqrt{2 / \nu}$.

$A_{b}(c \rightarrow \infty)=0$. Thus, in contrast to the case $b=1$, there is no phase transition as a function of $c$ in the sense of [14]. For $b=1$ the solution of (16) is $A_{1}(c)=1-c / a$ which reflects the phase transition at $c=a$ and is confirmed by the exact solution presented in [14].

In order to self-consistently check the validity of the MFA we need to investigate the behavior of $V_{l}$, the variance of $Q_{l}(x)$. Specifically, the MFA is justified if it can be shown that $V_{l} / z_{l}^{2} \rightarrow 0$ as $l \rightarrow \infty$. The detailed analysis in Appendix B shows that this is indeed the case. Moreover, although we only used the specific form $1-F_{g}(x)=e^{-x^{\alpha} / a}$, the above conclusions about the leading asymptotic behavior remain valid as long as $-\ln [1-F(x)]=-x^{\alpha} / a+o\left(x^{\alpha}\right)$, that is, the asymptotic behavior is universal in that the leading behavior of $-\ln [1-F(x)]$ determines the behavior of $\delta$-exceedance record values.

\subsection{Weibull class}

Now we consider $F_{w}(x)$. It turns out that it is possible to find the generating function

$$
\mathcal{G}_{l}(\lambda)=\int_{0}^{r} e^{\lambda(x-r)} Q_{l}(x) d x=e^{-r \lambda} G_{l}(-\lambda),
$$

where $G_{l}$ is defined in (A.1). From (A.11) with $1 / h_{w}(x)=\nu(r-x)$, we obtain the recursion relation for $\mathcal{G}_{l}$ in the asymptotic regime as

$$
\mathcal{G}_{l+1}=e^{-\lambda \delta_{l}} \mathcal{G}_{l}-\nu \lambda \frac{d \mathcal{G}_{l+1}}{d \lambda},
$$


where we neglect the contribution of the integral in the domain $\left(0, \delta_{l}\right)$. If we assume $\mathcal{G}_{l+1} \approx \mathcal{G}_{l}$, (24) becomes a first order differential equation

$$
\nu \lambda \frac{d \mathcal{G}_{l}}{d \lambda}+\left(1-e^{-\lambda \delta_{l}}\right) \mathcal{G}_{l}=0
$$

with the 'initial' condition $\mathcal{G}_{l}(\lambda=0)=1$. Within this approximation scheme, we get

$$
\ln \mathcal{G}_{l} \approx-\frac{1}{\nu} \operatorname{Ein}\left(\lambda \delta_{l}\right)
$$

where

$$
\operatorname{Ein}(x) \equiv \int_{0}^{x} \frac{1-e^{-t}}{t} d t=-\sum_{n=1}^{\infty} \frac{(-x)^{n}}{n ! n},
$$

which is an entire function (this function is also found in Ref. [17]). Since $\ln \mathcal{G}_{l}$ is the generating function of cumulants, the $n$ 'th cumulant is equal to $\delta_{l}^{n} /(n \nu)$. For example,

$$
\zeta_{l} \equiv r-z_{l}=\frac{\delta_{l}}{\nu}, \quad \sigma_{l}=\frac{\delta_{l}}{\sqrt{2 \nu}}
$$

In figure 2, we present simulation results for $b=\frac{1}{2}$ and for various $\nu$, observing an excellent agreement with (28).

We conclude that in this case the approach of the mean record value $z_{l}$ to the upper boundary $r$ is completely determined by the behavior of the handicaps. The approach is algebraic rather than exponential as in the standard case, confirming our expectation that the handicaps dominate the record statistics for any $c>0$ when the background process belongs to the Weibull class.

The full distribution of record values $Q_{l}(x)$ can be obtained by inverse Laplace transformation of $\mathcal{G}_{l}$ such that

$$
Q_{l}(r-x)=\frac{1}{2 \pi i} \int_{-i \infty}^{i \infty} \exp \left[\lambda x-\frac{1}{\nu} \operatorname{Ein}\left(\lambda \delta_{l}\right)\right] d \lambda=\frac{1}{\delta_{l}} g\left(x / \delta_{l}\right),
$$

where

$$
g(z)=\frac{1}{2 \pi i} \int_{-i \infty}^{i \infty} \exp \left[t z-\frac{1}{\nu} \operatorname{Ein}(t)\right] d t .
$$

Since $\operatorname{Ein}(t) \sim \ln |t|$ for $|t| \gg 1(|\operatorname{Arg}(t)|<\pi)$, we find $\mathcal{G}_{l}(\lambda) \approx\left(\lambda \delta_{l}\right)^{-1 / \nu}$ for $\lambda \delta_{l} \gg 1$, which implies that $Q_{l}(r-x)$ behaves as $x^{1 / \nu-1}$ for small $x$, just like $f_{w}(x)$.

\subsection{Fréchet class}

Now we consider $F_{f}(x)$ with $1 / h_{f}(x)=(a+x) / \mu$. Neglecting the contribution from the integral over the domain $\left(0, \delta_{l}\right)$, we get the recursion relation of $z_{l}$ for large $l$ as

$$
\left(1-\frac{1}{\mu}\right)\left(z_{l+1}+a\right) \approx\left(z_{l}+a\right)-\delta_{l}
$$

whose solution is

$$
z_{l}+a=\left(\frac{\mu}{\mu-1}\right)^{l}\left(z_{0}+a\right)+\sum_{k=0}^{l-1}\left(\frac{\mu}{\mu-1}\right)^{l-k} \delta_{k} \approx\left(\frac{\mu}{\mu-1}\right)^{l}\left(z_{0}+a+C_{0}\right),
$$


where $C_{0}=\sum_{k=0}^{\infty}(1-1 / \mu)^{k} \delta_{k}$. The above solution is exact when $c=0$. Recall that $\mu$ should be larger than 1 in order to have a finite mean $z_{l}$. The exponential growth of $z_{l}$ is identical to the known result for the i.i.d. record process [2]. We conclude that the asymptotics is not affected by $\delta_{l}$ if $b<1$ and the background process belongs to the Fréchet class.

\section{Increasing handicap}

This section analyzes the case $b>1$. A trivial conclusion for distributions with bounded support such as $F_{w}$ is immediate: As soon as $\delta_{l}>r$, all random variables are records. The effects for unbounded distributions are more subtle and will be discussed in the following, mostly focusing on the Gumbel class distributions $F_{g}$.

\subsection{Mean field analysis for the Gumbel class}

As in the previous section, we first look for a solution with diverging $z_{l}$, assuming $Q_{l}(x) \approx \delta\left(x-z_{l}\right)$. The recursion relation for $z_{l}$ under the MFA is

$$
z_{l+1}-z_{l}=\frac{a}{\alpha} z_{l+1}^{1-\alpha}-\delta_{l}+\left(\delta_{l}-z_{l}\right) \Theta\left(\delta_{l}-z_{l}\right)
$$

where $\Theta$ is the Heaviside step function with $\Theta(x)=1$ if $x>0$ and 0 otherwise. Since $\delta_{l}$ also diverges with $l$, we cannot simply neglect the integral over the domain $\left(0, \delta_{l}\right)$. If $\delta_{l}>z_{l}$, the above recursion has the solution

$$
z_{l}=(a / \alpha)^{1 / \alpha}
$$

which is of the order of the mean of $f_{g}$ and does not depend on $l$. This shows that it is not possible to have a diverging solution that increases more slowly than the handicaps for $b>1$. Furthermore, if $z_{l}$ saturates to a finite number, $Q_{l}(x)$ cannot be approximated as a $\delta$-function and the MFA does not apply. Rather, if $z_{l} \ll \delta_{l}$ all events would asymptotically be records with probability 1 and $Q_{l}(y) \rightarrow f(y)$ as $l \rightarrow \infty$. We will return to this scenario in the next subsection.

For now, let us assume that $z_{l}>\delta_{l}$ and ask when a diverging solution can exist. We start from (12) with $b>1$. If $\alpha \geq 1$, diverging $z_{l}$ implies $z_{l}^{1-\alpha} \ll \delta_{l}$, which leads to the contradictory relation $z_{l+1}<z_{l}$. Thus, for $\alpha \geq 1$ no diverging solution is possible and asymptotically all random variables in the background process are records.

The analysis for the case $\alpha<1$ is similar to that in section 3.1, except that now $b>1$. Assuming $z_{l}^{\alpha} \approx a(A l)^{\gamma}$, we arrive at (14). By the same reasoning as in section 3.1, $\gamma$ cannot be larger than 1 . If $\gamma=1$, we have either $\alpha<1 / b$ with $A=1$ or $\alpha=1 / b$ with $A$ being the solution of (16) with $b>1$. In contrast to the case $b<1$, however, (16) for $b>1$ does not have a positive solution if $c$ is larger than a 'threshold'

$$
c_{t}=a^{b}\left(1-b^{-1}\right)^{b-1} \text {. }
$$

Thus we expect that $z_{l}$ diverges as $l^{1 / \alpha}$ for $c<c_{t}$ and saturates to a finite value for $c>c_{t}$. On the other hand, assuming $\gamma<1$ as in section 3.1, we get $\gamma=(1-b) /(1-1 / \alpha)$. 
However, the condition $\gamma<1$ implies $\alpha<1 / b$, and we have seen above that for this case a solution with $\gamma=1$ exists. Hence the solution with $\gamma<1$ can at best describe the subleading behavior.

To summarize, the asymptotic behavior of $z_{l}$ as predicted by the MFA for $\alpha \neq 1 / b$ is,

$$
\frac{z_{l}^{\alpha}}{a}= \begin{cases}l-o(l), & \alpha<1 / b, \\ \text { finite, } & \alpha>1 / b,\end{cases}
$$

and for $\alpha=1 / b$,

$$
\frac{z_{l}^{\alpha}}{a}= \begin{cases}A_{b}(c) l-o(l), & c \leq c_{t} \\ \text { finite, } & c>c_{t} .\end{cases}
$$

\subsection{Stochastic bistability}

The value of the amplitude $A_{b}(c)$ in (37) is finite at $c=c_{t}$, which is suggestive of a first order phase transition as a function of $c$. We will see in this subsection that such a transition indeed exists, but its character is importantly modified by fluctuations that have been neglected in the MFA. To make the point clear, we limit ourselves to the distribution $F_{g}(x)=1-\exp \left(-x^{1 / b}\right)$ with $a=1$ which was anticipated to exhibit a phase transition at $c=c_{t}$. As before, we consider only the asymptotic regime.

Suppose that the $l$ 'th record happens to be smaller than $\delta_{l}, Y_{l}<\delta_{l}$. As a consequence of the definition (4) and the fact that the support of the distribution $F(x)$ is limited to the positive real line, the next background event following the l'th record is then a record with probability 1 . Since its value $Y_{l+1}$ is an unconstrained draw from the background distribution, the probability that $Y_{l+1}$ is larger than $\delta_{l+1}$ is

$$
P_{l+1}^{>}=1-F_{g}\left(\delta_{l+1}\right) \approx \exp \left(-c^{1 / b} l^{(b-1) / b}\right),
$$

which is very small for large $l$. Thus we see that the process is effectively trapped in the state $Y_{k}<\delta_{k}$, where all events are 'records' drawn from the background distribution. As $\delta_{k}$ increases with $k$, the corresponding probability $P_{k}^{>}$decreases further for $k>l+1$, and the expected time until the process for the first time reverts to $Y_{k}>\delta_{k}$ is larger than $1 / P_{l+1}^{>} \sim \exp \left(c^{1 / b} l^{(b-1) / b}\right) \gg l$. On the other hand, if $Y_{l} \sim z_{l} \sim l^{b}$ for sufficiently large $l$ as predicted by the MFA in section 4.1, $Y_{l}$ remains larger than $\delta_{l}$ with high probability because $\delta_{l}$ only increases as $l^{b-1}$.

We conclude that the sample paths of the process segregate into two subpopulations, a stationary population in which all events of the background process are records and a diverging population where $Y_{l}$ grows more rapidly than $\delta_{l}$. Figure 3 shows a realization of the segregation phenomenon due to initial fluctuations.

To account for this behavior, we approximate the distribution $Q_{l}(x)$ by a sum of two contributions,

$$
Q_{l}(x) \approx Q^{(s)} f_{g}(x)+\left(1-Q^{(s)}\right) \delta\left(x-\tilde{z}_{l}\right),
$$




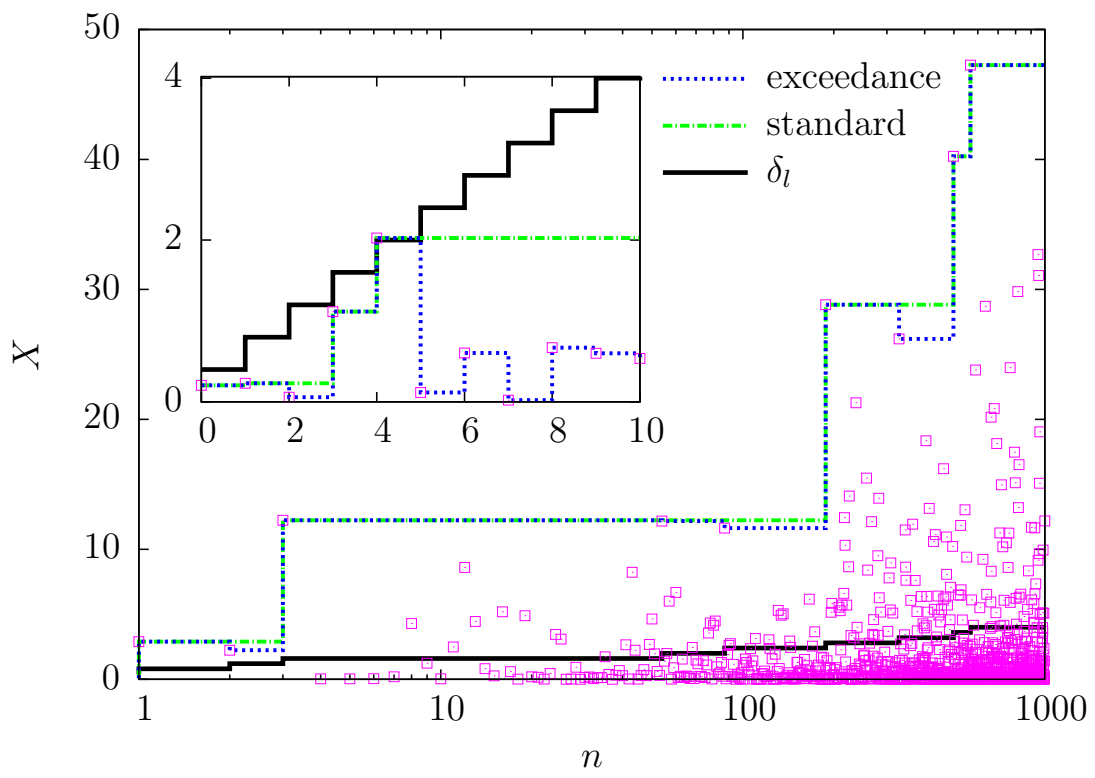

Figure 3. A sample path of $\delta$-exceedance records (blue dotted line) for $b=1 / \alpha=2$ and $c=0.4$ on a semi-logarithmic scale. There are 9 new records in the figure. For comparison, the corresponding standard records (green dot-dashed line) and the handicaps $\delta_{l}$ (black solid line) are also drawn. The random variables of the background process are represented by open squares. In this case, the $\delta$-exceedance records are very close to the standard records. Inset: Another sample path for the same parameter sets. In this path, the $\delta$-exceedance records are smaller than $\delta_{l}$. As in the main figure, there are $9 \delta$-exceedance records which however occur on a much shorter time scale $(n=9$, all events are records).

where $\tilde{z}_{l}$ should diverge faster than $\delta_{l}$ and $Q^{(s)}$ is the limiting value of the probability $Q_{l}^{(s)}$ that $Y_{l}$ is smaller than $\delta_{l}$,

$$
Q^{(s)}=\lim _{l \rightarrow \infty} Q_{l}^{(s)} \equiv \lim _{l \rightarrow \infty} \mathbb{P}\left[Y_{l}<\delta_{l}\right] .
$$

This quantity measures the relative weight of the stationary subpopulation of sample paths and will serve as an order parameter for the phase transition in the following.

Plugging (39) into (11) and keeping the leading terms, we get

$$
\tilde{z}_{l+1}-\tilde{z}_{l}=\frac{a}{\alpha} \tilde{z}_{l+1}^{1-\alpha}-\delta_{l},
$$

where we approximate $\int_{0}^{\delta_{l}}\left(\delta_{l}-x\right) Q_{l}(x) d x \approx Q^{(s)} \delta_{l}$. Hence, the diverging solution found in section 4.1 actually describes the behavior of $\tilde{z}_{l}$. Note that the mean $z_{l}$ and standard deviation $\sigma_{l}$ of $Q_{l}(x)$ are related to $\tilde{z}_{l}$ by $\left(0<Q^{(s)}<1\right)$

$$
z_{l} \approx \tilde{z}_{l}\left(1-Q^{(s)}\right), \quad \sigma_{l} \approx \tilde{z}_{l} \sqrt{Q^{(s)}\left(1-Q^{(s)}\right)}
$$

which yields the relation

$$
\frac{z_{l}}{\sigma_{l}} \approx \sqrt{\frac{1}{Q_{l}^{(s)}}-1} \equiv R_{l} .
$$

In this context, the absence of a solution for the prefactor $A$ for $c>c_{t}$ can be interpreted as $Q^{(s)}=1$. Since the prefactor $A$ at $c=c_{t}$ is $A^{*}=\left(1-b^{-1}\right)^{b} a^{b}$, we expect that $Q^{(s)}$ 


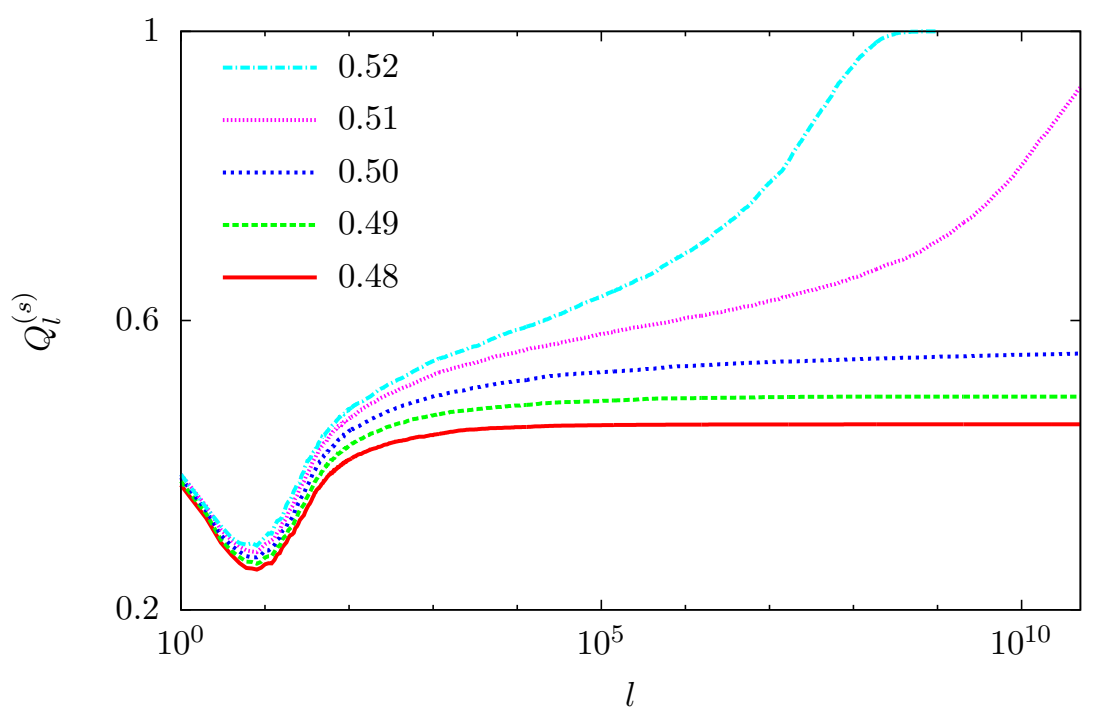

Figure 4. Semi-logarithmic plot of $Q_{l}^{(s)}$ vs. $l$ for $b=1 / \alpha=2$ and $\delta_{l}=c(l+1)$ for $c$ around the threshold value $c_{t}=\frac{1}{2}$.

is strictly smaller than 1 even at $c=c_{t}$. Hence we can conclude that a discontinuous transition in terms of $Q^{(s)}$ occurs at $c=c_{t}$.

To support the above theory, we numerically studied the case with $b=1 / \alpha=2$. For this case, $c_{t}=\frac{1}{2}$ and

$$
A=\frac{1+\sqrt{1-2 c}}{2}
$$

which is the solution of $A-A^{2}=c / 2$. Note that we have only taken the larger solution, expecting that $A$ is a continuous function of $c$ for $0 \leq c<c_{t}$. We first check if $Q^{(s)}$ shows a discontinuity at the threshold value $c_{t}=\frac{1}{2}$. In figure 4, $Q_{l}^{(s)}$ is depicted as a function of $l$ for $c$ around the threshold value. Each curve is the result of $10^{4}$ independent runs. As anticipated, there is a clear indication of a discontinuous jump at $c=c_{t}=\frac{1}{2}$.

Next, we check if (39) is a valid assumption by comparing $z_{l} / \sigma_{l}$ with $R_{l}$ in figure 5 , The asymptotic behaviors of both quantities are indeed in good agreement with each other. The inset of figure 5 compares $\tilde{z}_{l} \equiv z_{l} /\left(1-Q_{l}^{(s)}\right)$ with the anticipated asymptotic behavior $(A l)^{2}$ with $A$ given by (44). This also shows an excellent agreement. Although we only present data for $c=0.2$, similar agreement is observed in a wide range of $c$ for $c \leq \frac{1}{2}$.

As can be seen from figure 5, $R_{l}$ saturates in a rather short time. This indicates that the asymptotic behavior of $Q_{l}^{(s)}$ is almost determined by the fluctuation of $Y_{l}$ when $l$ is small; see also figure 3. That is, the analysis of the asymptotic behavior of $Q_{l}(x)$ and corresponding quantities cannot give much information about $Q^{(s)}$, and we do not think $Q^{(s)}$ is universal in the sense that it is only determined by the leading behavior of $\delta_{l}$. For example, if $\delta_{l}=c\left(l+l_{0}\right)^{b-1}$ with very large $l_{0}, \delta_{l}$ for small $l$ is at least $c l_{0}^{b-1}$ which means $Q_{l}^{(s)}$ is almost 1 for any $\alpha$. Hence, we have to resort to numerical analysis to find $Q^{(s)}$. 


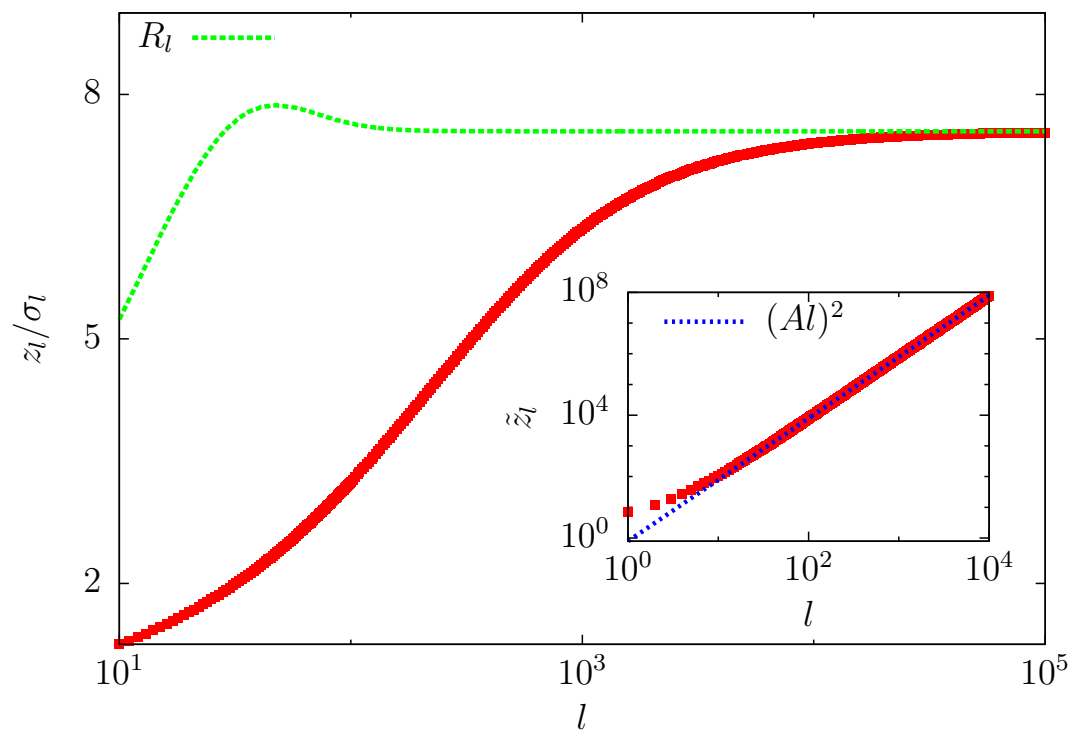

Figure 5. Semi-logarithmic plot of $z_{l} / \sigma_{l}$ (symbols) vs. $l$ for $b=1 / \alpha=2$ and $\delta_{l}=c(l+1)$ with $c=0.2$. For comparison, $R_{l}$ defined in (43) is also drawn (top curve). Inset: Double logarithmic plot of $\tilde{z}_{l} \equiv z_{l} /\left(1-Q_{l}^{(s)}\right)$ (symbols) vs. $l$ for $b=1 / \alpha=2$ and $c=0.2$. The straight line is a plot of $(A l)^{2}$ against $l$ with $A$ given by (44).

Since $Q^{(s)}=0$ when $c=0$, it is an interesting question how $Q^{(s)}$ approaches zero as $c \rightarrow 0$. We investigated the behavior of $Q^{(s)}$ for small $c$ via simulations. As figure 6 shows, $Q^{(s)}$ decreases quite fast for small $c$, which suggests a form

$$
Q^{(s)}(c) \sim \chi_{1} \exp \left(-\chi_{2} / c\right)
$$

with two parameters $\chi_{1}$ and $\chi_{2}$. If this is the case, a plot of $-\ln Q^{(s)}$ as a function of $1 / c$ should be well fitted by a straight line. Indeed, as the inset of figure 6 shows, a linear function well approximates the data with parameter values $\ln \chi_{1} \approx 1.9$ and $\chi_{2} \approx 1.15$.

It should be clear from the above discussion that the stochastic bistability scenario with a nonzero $Q^{(s)}$ is not restricted to $\alpha=1 / b$ but should apply also for $\alpha<1 / b$, where the MFA predicts standard record behavior with $z_{l}^{\alpha} \approx a$ l. In contrast to the case $\alpha=1 / b$, however, for $\alpha<1 / b$ we expect $Q^{(s)}$ to be smoothly increasing function of $c$ that approaches unity only asymptotically for large $c$. We have checked numerically that this is indeed the case, and found that the behavior of $Q^{(s)}$ for small $c$ is again well described by the functional form (45).

\subsection{Fréchet class}

For $Q^{(s)}$ to remain zero for $c>0$, the probability that $Y_{l}>\delta_{l}$ under the condition that $Y_{l-1}<\delta_{l-1}$ should not be negligibly small for large $l$. For the Gumbel (and Weibull) classes, this scenario is clearly not feasible as we have seen and only the Fréchet class might allow for such a possibility. Let us consider $F_{f}(x)$ with $a=1$. The probability of interest is $P_{l}^{>} \sim l^{-\mu(b-1)}$, hence such an event would happen after $m \sim l^{\mu(b-1)}$ records. If 


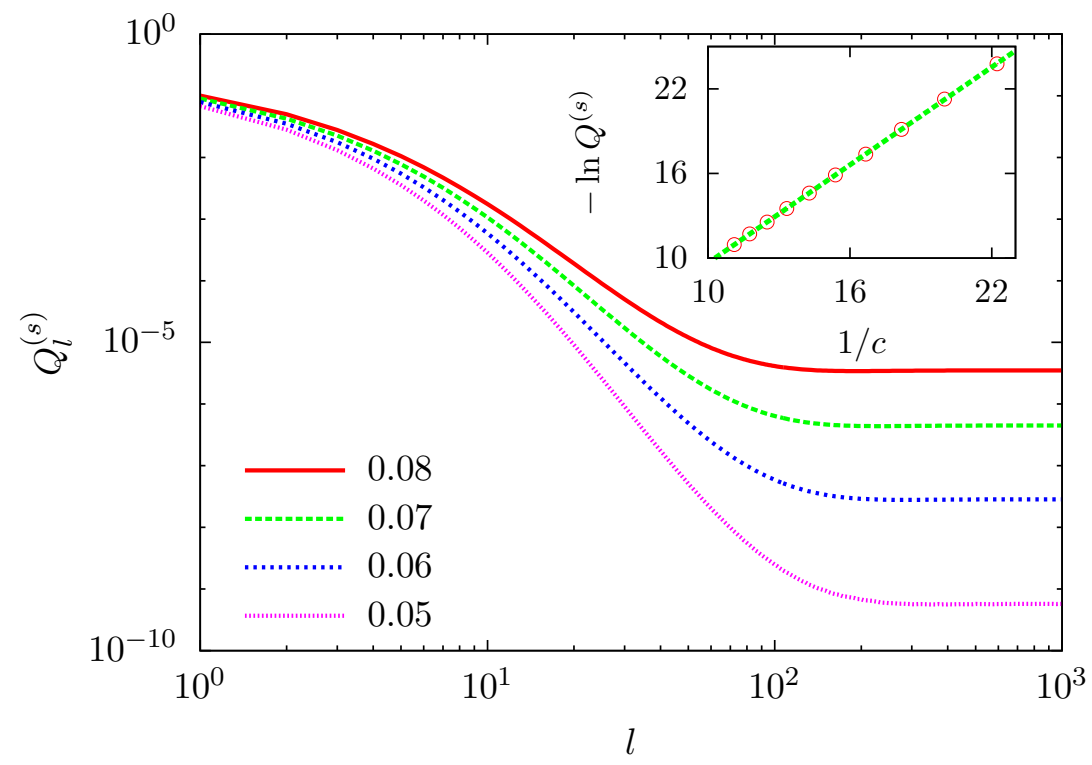

Figure 6. Plots of $Q_{l}^{(s)}$ vs. $l$ for various values of $c$ on a double-logarithmic scale. Inset: Plot of $-\ln Q^{(s)}$ vs. $1 / c$. The straight line is the result of a linear fit. The number of independent runs for each data set is $2.5 \times 10^{12}$.

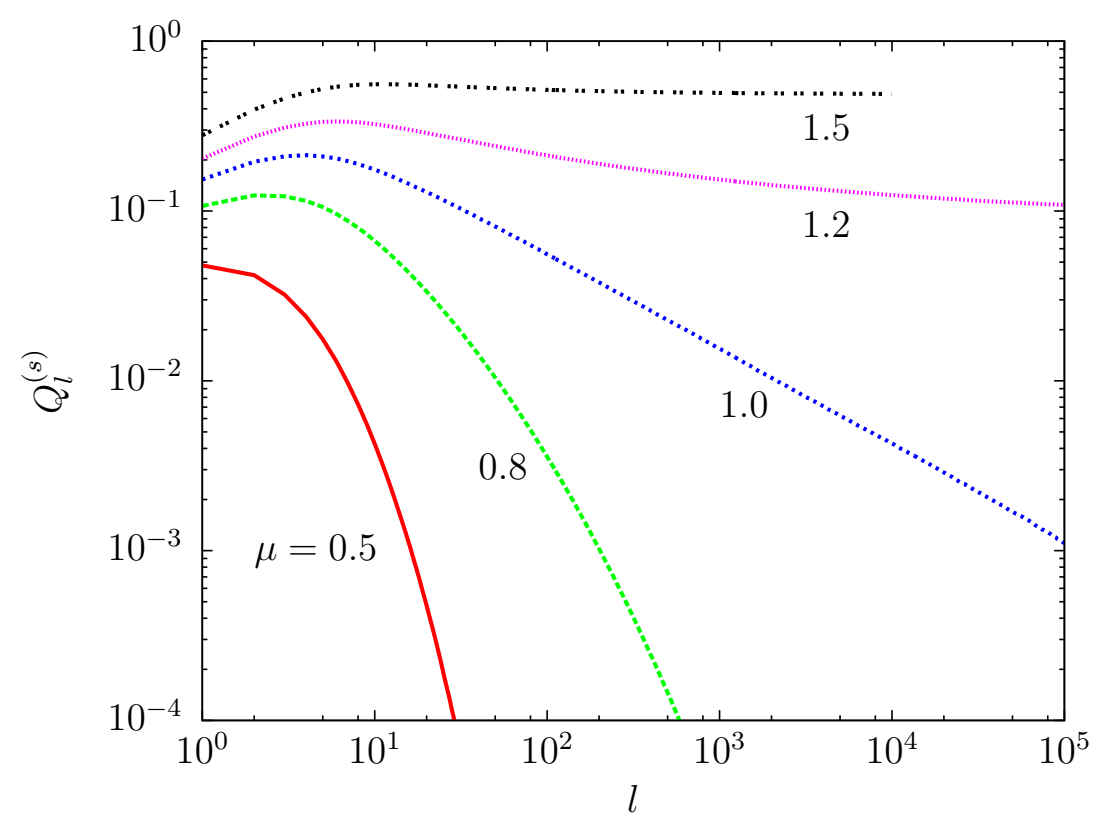

Figure 7. Plots of $Q_{l}^{(s)}$ vs. $l$ for $b=2$ and $c=1$ on a double-logarithmic scale for Fréchet class distributions. The values of the power law exponent $\mu$ in this figure are $0.5,0.8,1.0,1.2$, and 1.5 (bottom to top), respectively. As predicted by the theory, $Q_{l}^{(s)}$ saturates to a finite value when $\mu>1 /(b-1)=1$.

$\mu(b-1)<1, l \gg m$ for sufficiently large $l$ and the process can escape from the stationary regime before $P_{l}^{>}$changes substantially. Thus, we expect $Q^{(s)}=0$ if $\mu<1 /(b-1)$.

To confirm this argument, we performed simulations for $b=2$ and various $\mu$. For convenience, we fix $c=1$. Figure 7 depicts $Q_{l}^{(s)}$ as a function of $l$ for $\mu=0.5,0.8$, 
1.0, 1.2, and 1.5. As anticipated, the long time behavior of $Q_{l}^{(s)}$ changes qualitatively at $\mu_{c}=1 /(b-1)=1$. As a final remark, $Q_{l}^{(s)}$ seems to exhibit a power-law decay at $\mu=\mu_{c}$ with a power close to 0.5 .

\section{Length of adaptive walks}

Using the results of the previous sections, we analyze the length of adaptive walks when a population starts from a low fitness genotype $\mathcal{C}_{0}$. As was extensively discussed in Ref. [14], we can assume that every step increases the mutational distance to $\mathcal{C}_{0}$, and that all possible neighboring genotypes of higher fitness from genotype $\mathcal{C}_{k}$ are located in the 'forward' direction at distance $k+1$. In the initial state $\mathcal{C}_{0}$ the number of forward neighbors is $L$, and after $l$ steps it reduces to $L-l$. Let $Q_{l}(y, L)$ denote the probability density that the random part, i.e., $\eta_{\mathcal{C}_{l}}$ in (2) is $y$ provided that the walker takes at least $l$ steps. Then the recursion relation for $Q_{l}(y, L)$ is found to be

$$
Q_{l+1}(y, L)=f(y) \int_{-\infty}^{y+\delta_{l}} Q_{l}(x, L) \frac{1-F\left(x-\delta_{l}\right)^{L-l}}{1-F\left(x-\delta_{l}\right)} d x,
$$

where the term $F\left(x-\delta_{l}\right)^{L-l}$ accounts for the possibility that the walker stops because none of the $L-l$ forward neighbors is of higher fitness [14]. This term is absent in the corresponding recursion relation (7) for the distribution $Q_{l}(x)$ of the $\delta$-exceedance record process, and hence the two distributions are related by

$$
Q_{l}(x)=\lim _{L \rightarrow \infty} Q_{l}(x, L) .
$$

Denoting by $H_{l}$ and $P_{l}$ the probability that the walker takes at least $l$ steps and that the walker stops at the l'th step, respectively, we can write

$$
\begin{aligned}
& H_{l}=\int_{-\infty}^{\infty} Q_{l}(x, L) d x \\
& P_{l} \equiv H_{l}-H_{l+1}=\int_{-\infty}^{\infty} Q_{l}(x, L) F\left(x-\delta_{l}\right)^{L-l} d x,
\end{aligned}
$$

which are used to calculate the mean walk distance

$$
D_{\mathrm{RAW}}=\sum_{l=0}^{L} l P_{l}
$$

\subsection{Gumbel class}

In this subsection, we will calculate $D_{\text {RAW }}$ for the Gumbel class using the results of section 3 and section 4 . When $b>1$, we have shown that the record process gets trapped in a state where all entries are records with a non-zero probability $Q^{(s)}$. For the adaptive walk this means that every randomly chosen neighboring genotype in the forward direction is of higher fitness, and the walk therefore attains the maximal possible length $l=L$. On the other hand, with probability $1-Q^{(s)}$ the walk behaves similar to the case $b<1$ where, as we will show below, the walk length increases only logarithmically 
with $L$. Thus the distribution of walk lengths for $b>1$ is bimodal, with the mean walk length being dominated by the peak at $l=L$ and hence $D_{\mathrm{RAW}} \sim O(L)$.

To calculate $D_{\text {RAW }}$ with $b<1$, we employ the following approximation scheme. Since $Q_{l}(x)$ can be understood as the density of the random part at the l'th step conditioned on the walker taking at least $l$ steps irrespective of $L$, we approximate $Q_{l}(x, L)$ as $H_{l} Q_{l}(x)$, which in turn gives

$$
P_{l} \approx H_{l} \int_{-\infty}^{\infty} Q_{l}(x) F\left(x-\delta_{l}\right)^{L-l} d x .
$$

In particular, when $Q_{l}(x) \approx \delta\left(x-z_{l}\right), P_{l}$ becomes

$$
P_{l}=H_{l}-H_{l+1}=H_{l} F\left(z_{l}-\delta_{l}\right)^{L-l} .
$$

We have shown in section 3 that $F\left(z_{l}-\delta_{l}\right)$ can generally be approximated as $\exp \left[-e^{-(A l)^{\gamma}}\right]$, with certain numbers $A>0$ and $\gamma \leq 1$. Since $H_{l}$ is not expected to be significantly different from $H_{l+1}$, we can treat $H_{l}$ as a differentiable function of $l$. Hence, for sufficiently large $l$ and $L \gg l$ we get

$$
\frac{d H(l)}{d l}=-H(l) \exp \left[-L e^{-(A l)^{\gamma}}\right]
$$

with the solution

$$
H(l) \approx \exp \left[-\int_{0}^{l} \widetilde{P}(x) d x\right]
$$

where

$$
\widetilde{P}(x)=\exp \left[-L e^{-(A x)^{\gamma}}\right] .
$$

Since $P_{l} \approx-\frac{d H}{d l}$, we arrive at

$$
D_{\mathrm{RAW}} \approx \int_{0}^{\infty} y \widetilde{P}(y) \exp \left[-\int_{0}^{y} \widetilde{P}(x) d x\right] d y
$$

where we have assumed that $P_{l}$ is negligible if $l=O(L)$.

By the change of variables $y=w+(\ln L)^{1 / \gamma} / A$ and $x=z+(\ln L)^{1 / \gamma} / A$, we get

$D_{\mathrm{RAW}} \approx \int_{-\infty}^{\infty} d w\left[\frac{(\ln L)^{1 / \gamma}}{A}+w\right] \exp \left[-e^{-w / K}-\int_{-\infty}^{w} \exp \left[-e^{-z / K}\right] d z\right]$,

where $K=(\ln L)^{1 / \gamma-1} /(A \gamma)$. After further substitutions $e^{-w / K}=x$ and $e^{-z / K}=t$, we obtain

$$
D_{\mathrm{RAW}} \approx \int_{0}^{\infty} d x\left[\frac{(\ln L)^{1 / \gamma}}{A}-K \ln x\right] \frac{d}{d x} \exp \left[-K E_{1}(x)\right]
$$

where

$$
E_{1}(x)=\int_{x}^{\infty} \frac{e^{-t}}{t} d t
$$

is the exponential integral function. Thus, we have

$$
D_{\mathrm{RAW}} \approx \frac{(\ln L)^{1 / \gamma}}{A}+\frac{1}{A \gamma}(\ln L)^{1 / \gamma-1} \mathcal{K}(K),
$$



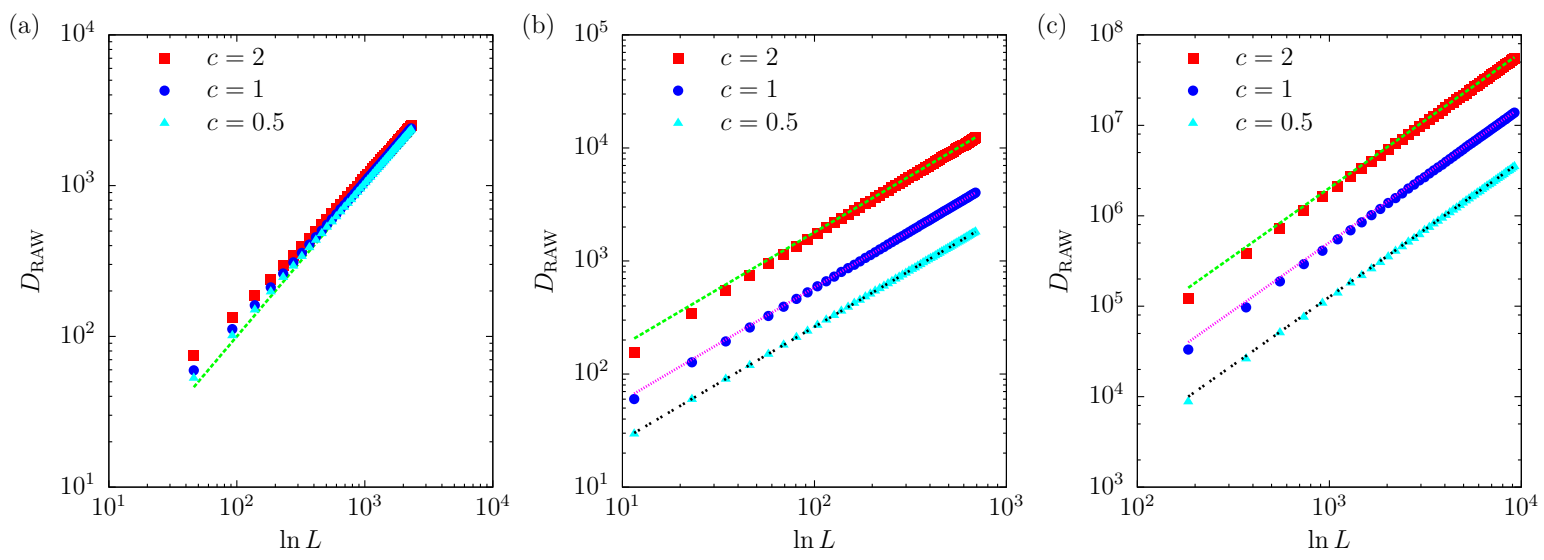

Figure 8. Double-logarithmic plots of the adaptive walk length $D_{\mathrm{RAW}}$ vs. $\ln L$ for $b=\frac{1}{2}$ and Gumbel-class random variables with (a) $\alpha=1$, (b) $\alpha=2$, and (c) $\alpha=4$. Straight lines are the predicted leading term in (60).

where

$$
\mathcal{K}(K) \equiv-\int_{0}^{\infty} \ln x \frac{d}{d x} \exp \left[-K E_{1}(x)\right] d x
$$

For $A=\gamma=1$ the numerical value of $\mathcal{K}(K)$ is 1.099124 which coincides with the exact result for $c=0$ [17]. However, since our analysis neglects the effect of fluctuations, the subleading behavior of (60) cannot generally be expected to be exact. The leading order behavior $D_{\mathrm{RAW}} \approx(\ln L)^{1 / \gamma} / A$ with $A$ and $\gamma$ given in (21) and (22), respectively, is compared to simulations in figure 8 , showing excellent agreement.

\subsection{Weibull class}

Now we consider the bounded distribution of Weibull type $F_{w}$. Since the case $b>1$ is trivial, we limit ourselves to the case $b<1$. For a rough estimate of the adaptive walk length, we ask at which value of $l$ the stopping probability $F\left(x-\delta_{l}\right)^{L-l}$ in (46) has an appreciable magnitude when evaluated near the mean record value $z_{l}$ [14]. We have seen in section 3.2 that the approach of $z_{l}$ towards the upper boundary $r$ is determined by the behavior of the handicaps, in the sense that $r-z_{l} \sim \delta_{l}$. Thus using (28) we have

$$
F_{w}\left(z_{l}-\delta_{l}\right)^{L-l} \approx \exp \left[-\left(\frac{(1+\nu) c}{\nu r}\right)^{1 / \nu} \frac{L-l}{l^{(1-b) / \nu}}\right] .
$$

Let us first assume that $l \sim L^{\xi}$ for some $\xi<1$. If $1-b>\nu$ the right hand side of (62) becomes of order unity for $L \rightarrow \infty$ if $\xi=\nu /(1-b)$, leading to the prediction that

$$
D_{\mathrm{RAW}} \sim L^{\nu /(1-b)} .
$$

On the other hand, if $1-b<\nu$ the right hand side will vanish with $L \rightarrow \infty$ for any $\xi<1$, which implies that the walk length must be $O(L)$ to leading order. Indeed, taking $L-l=L^{\xi^{\prime}}$ we see that (62) approaches a nonzero limit if $\xi^{\prime}=(1-b) / \nu$, and we conclude that $D_{\mathrm{RAW}}=L-O\left(L^{(1-b) / \nu}\right)$ for any $c>0$ once $\nu>1-b$. 
For the case of $1-b>\nu$ our simulations show a rather systematic, though small, deviation from the prediction (63), which indicates the need for a more careful analysis. To get a more accurate expression of $D_{\mathrm{RAW}}$ for $\nu<1-b$, we start from the approximation (51), which now takes the form

$$
\frac{P_{l}}{H_{l}} \approx \int_{0}^{r} d x F_{w}\left(r-x-\delta_{l}\right)^{L} Q_{l}(r-x) \approx \mathcal{P}\left(\frac{K}{l}\right)
$$

where $K=\left(c L^{\nu} / r\right)^{1 /(1-b)}$,

$$
\mathcal{P}(x)=\int_{0}^{\infty} d y \exp \left[-x^{(1-b) / \nu}(1+y)^{1 / \nu}\right] g(y) d y
$$

and the function $g(y)$ was defined in (30). Since we expect $D_{\mathrm{RAW}} \sim o(L)$, we dropped the $l$ in $F^{L-l}$.

Although we do not know an explicit form for $g(y)$, we can still obtain the asymptotic behavior of $\mathcal{P}$ for small and large $x$. When $x \ll 1$, we can approximate $\mathcal{P}(x)$ as

$$
\mathcal{P}(x) \approx 1-x^{(1-b) / \nu} \int_{0}^{\infty}(1+y)^{1 / \nu} g(y) d y .
$$

When $x \gg 1$, the integral will be dominated by small $y$, so we can approximate

$$
\begin{aligned}
\mathcal{P}(x) & \approx \exp \left[-x^{(1-b) / \nu}\right] \int_{0}^{\infty} d y \exp \left[-\frac{x^{(1-b) / \nu}}{\nu} y\right] g(y) \\
& \sim \exp \left[-x^{(1-b) / \nu}\right] x^{-(1-b) / \nu^{2}} \nu^{1 / \nu},
\end{aligned}
$$

where we have used that $\mathcal{G}_{l}\left(z / \delta_{l}\right) \sim z^{-1 / \nu}$ for large $z$ (see section 3.2).

Following the same line of reasoning as in section 5.1, we obtain the approximate formula

$$
\begin{aligned}
D_{\mathrm{RAW}} & \approx \int_{0}^{\infty} d y y \mathcal{P}\left(\frac{K}{y}\right) \exp \left[-\int_{0}^{y} \mathcal{P}\left(\frac{K}{x}\right) d x\right] \\
& =K \int_{0}^{\infty} \frac{d z}{z} \exp \left[-K \int_{z}^{\infty} \mathcal{F}(t) d t+\ln \{K \mathcal{F}(z)\}\right],
\end{aligned}
$$

for the walk length, where $\mathcal{F}(x)=\mathcal{P}(x) / x^{2}$. To estimate the integral, we look for the saddle point $z_{c}$ that maximizes the argument of the exponential function, which satisfies the equation

$$
0=\left.\frac{d}{d z}\left[K \int_{z}^{\infty} \mathcal{F}(t) d t-\ln \mathcal{F}(z)\right]\right|_{z=z_{c}}=-K \mathcal{F}\left(z_{c}\right)-\left.\frac{d \ln \mathcal{F}(z)}{d z}\right|_{z=z_{c}} .
$$

Since $K$ is large, $z_{c}$ should be either very small or very large. Let us assume that $z_{c}$ is very small. Approximation of $\mathcal{P}$ for small $x$ in (66) gives $\mathcal{F}(z) \sim z^{-2}$, which suggests $-K / z_{c}^{2}+2 / z_{c}=0$ or $z_{c} \sim K$. Thus, assuming that $z_{c}$ is very small leads to a contradiction.

Let us now investigate if a large $z_{c}$ solution exists. When $z$ is large, $\mathcal{F}(z) \approx$ $C_{0} z^{-v} \exp \left(-z^{\epsilon}\right)$ with $\epsilon=(1-b) / \nu, v=2+(1-b) / \nu^{2}$, and $C_{0}=\nu^{1 / \nu}$. Thus, we can approximate $\mathcal{F}^{\prime}\left(z_{c}\right) / \mathcal{F}\left(z_{c}\right) \approx-\epsilon z_{c}^{\epsilon-1}$ which, together with (69), gives

$$
K \mathcal{F}\left(z_{c}\right) \approx K C_{0} z_{c}^{-v} \exp \left(-z_{c}^{\epsilon}\right) \approx \epsilon z_{c}^{\epsilon-1}
$$




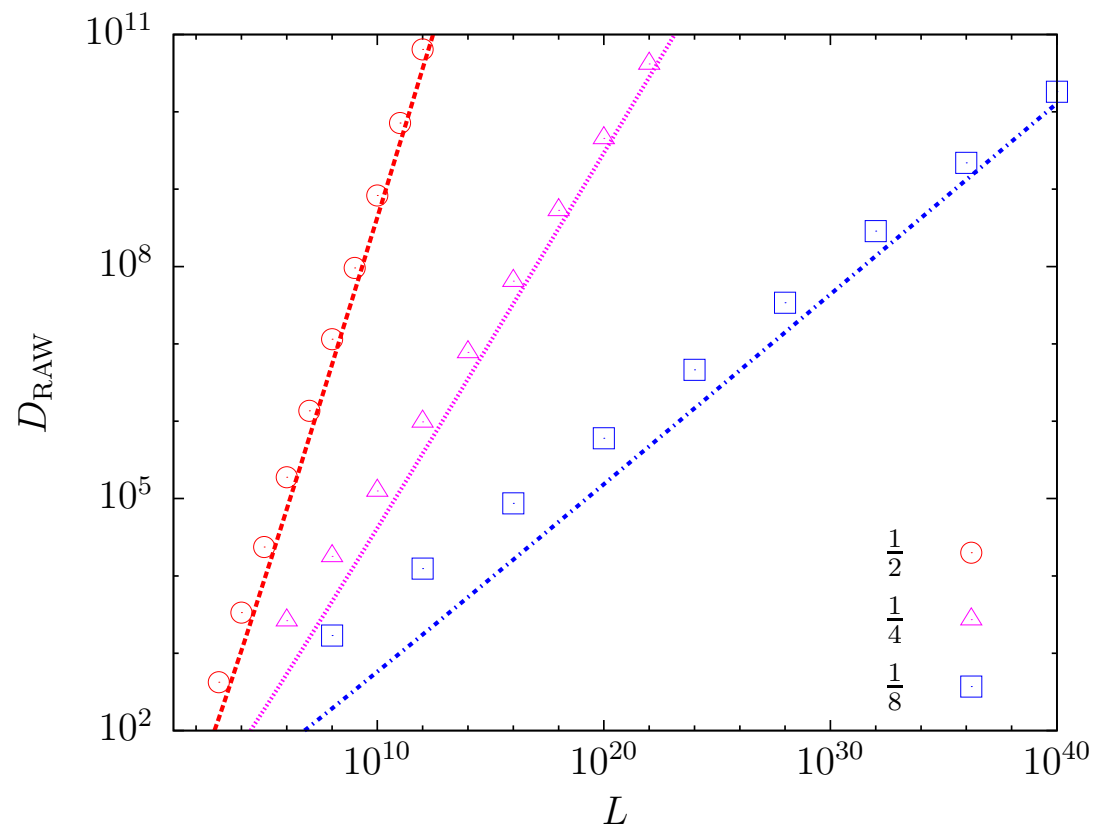

Figure 9. Double-logarithmic plots of the adaptive walk length $D_{\mathrm{RAW}}$ vs. $L$ for $b=\frac{1}{2}$ and distributions belonging to the Weibull class with $\nu=\frac{1}{2}$ (circles), $\frac{1}{4}$ (triangles), and $\frac{1}{8}$ (squares). For comparison, the prediction (73) is shown as straight lines.

Thus, we find the leading behavior of $z_{c}$ as

$$
z_{c} \sim(\ln K)^{\nu /(1-b)},
$$

which is consistent with the large $z_{c}$ assumption.

Using (71), we can now find $D_{\mathrm{RAW}}$. Since

$$
\exp \left[-K \int_{z}^{\infty} \mathcal{F}(t) d t+\ln \{K \mathcal{F}(z)\}\right]=\frac{d}{d z} \exp \left[-K \int_{z}^{\infty} \mathcal{F}(t) d t\right],
$$

and the exponential function is dominated by the region around $z=z_{c}$, we approximate

$$
\begin{aligned}
D_{\mathrm{RAW}} & \approx \frac{K}{z_{c}} \int_{0}^{\infty} d z \frac{d}{d z} \exp \left[-K \int_{z}^{\infty} \mathcal{F}(t) d t\right] \\
& =\frac{K}{z_{c}} \sim \frac{K}{(\ln K)^{\nu /(1-b)}}=\left(\frac{1-b}{\nu} \frac{L}{\ln L}\right)^{\nu /(1-b)}
\end{aligned}
$$

where we have used $\int_{0}^{\infty} \mathcal{F}(t) d t=\infty$ (recall that $\mathcal{F}(t) \sim t^{-2}$ for small $t$ ).

To confirm this asymptotic behavior, we performed numerical simulations for $b=\frac{1}{2}$. Figure 9 shows that the asymptotic behavior for large $L$ is well described by (73).

\section{Summary and conclusions}

In this paper we have investigated a modified record process defined on sequences of i.i.d. random variables in which the occurrence threshold for record events is reduced by a handicap $\delta_{k}$ that is a function of the record number $k$. This modification obviously increases the rate of record occurrence and decreases the magnitude of record values. 
However, similar to other cases where the standard record process is modified by rounding effects or trends [12, 10], the degree to which the record statistics are altered by the handicaps depends strongly on the tail properties of the background process. By allowing the handicaps to depend on the record number, we are able to tune their size to match the increments between the standard record values corresponding to a given background distribution. Building on the results obtained in [14], we have thus uncovered a rich variety of phase-transition like phenomena that emerge from the interplay of the stochastic record process with the deterministic handicap function $\delta_{k}$.

When the handicaps increase or decrease according to the power law (5) with exponent $b-1$, the distributions whose record increments match this behavior are the representatives $F_{g}$ of the Gumbel class with exponent $\alpha=1 / b$. In figure 10 we summarize our findings for this class of distributions in the form of a phase diagram in the $(\alpha, b)$-plane. There are four distinct regions separated by the lines $b=1 / \alpha$ and $b=1$. For $b>1$ (increasing handicap) we have seen that the sample paths of the record process display a kind of stochastic bistability, which leads to the decomposition of the distribution of record values $Q_{l}(x)$ into the general form

$$
Q_{l}(x)=Q^{(s)} \rho(x)+\left[1-Q^{(s)}\right] \tilde{\rho}_{l}\left(x-\tilde{z}_{l}\right) .
$$

Here $\rho(x)$ is a probability density with finite mean, $\tilde{z}_{l}$ diverges with $l$ and $\tilde{\rho}_{l}$ is a distribution with zero mean and a standard deviation that grows more slowly than $\tilde{z}_{l}$, such that the diverging part of (74) becomes concentrated around $\tilde{z}_{l}$ for large $l$. The emergence of a stationary component with weight $Q^{(s)}$ in a record process that by nature is non-stationary is perhaps the most remarkable feature of our work. It is well known that records from i.i.d. sequences with an added linear trend become asymptotically stationary [11], but the scenario of a first-order-like phase transition in the population of sample paths that we have described in section 4 does not appear to have any counterpart in previous studies of record processes.

In region I of the phase diagram $(b>1$ and $\alpha<1 / b), \tilde{z}_{l}^{\alpha}=l-o(l)$ and $Q^{(s)}$ is an increasing function of $c$ that satisfies $0<Q^{(s)}<1$ for all $c>0$. For small $c$ we have found that $Q^{(s)}$ is well approximated by the function (45) which displays an essential singularity at $c=0$. Region II $(b>1$ and $\alpha>1 / b)$ is characterized by $Q^{(s)}=1$, which means that almost all i.i.d. random variables become records when the number of record events is large. In both regions I and II, $\rho(x)$ in (74) is asymptotically equal to the density of the background process.

On the borderline between regions I and II which is depicted by the red solid curve in figure 10, $Q^{(s)}$ found to exhibit a discontinuous transition as $c$ increases. That is, there is a number $c_{t}>0$ such that $Q^{(s)}$ is strictly smaller than 1 if $c \leq c_{t}$ while $Q^{(s)}=1$ if $c>c_{t}$. When $c \leq c_{t}, \tilde{z}_{l}^{\alpha} \approx A_{b}(c) l$, where $A_{b}(c)$ is the (larger) positive solution of (16) with $b>1$. Again, along this curve $\rho(x)$ is the density of the background process when $Q^{(s)}>0$.

The behavior along the line $b=1$ (blue dot-dashed line in figure 10) was the topic of [14], but for completeness we include a discussion of this case in terms of $Q^{(s)}$. For $\alpha<1$, 


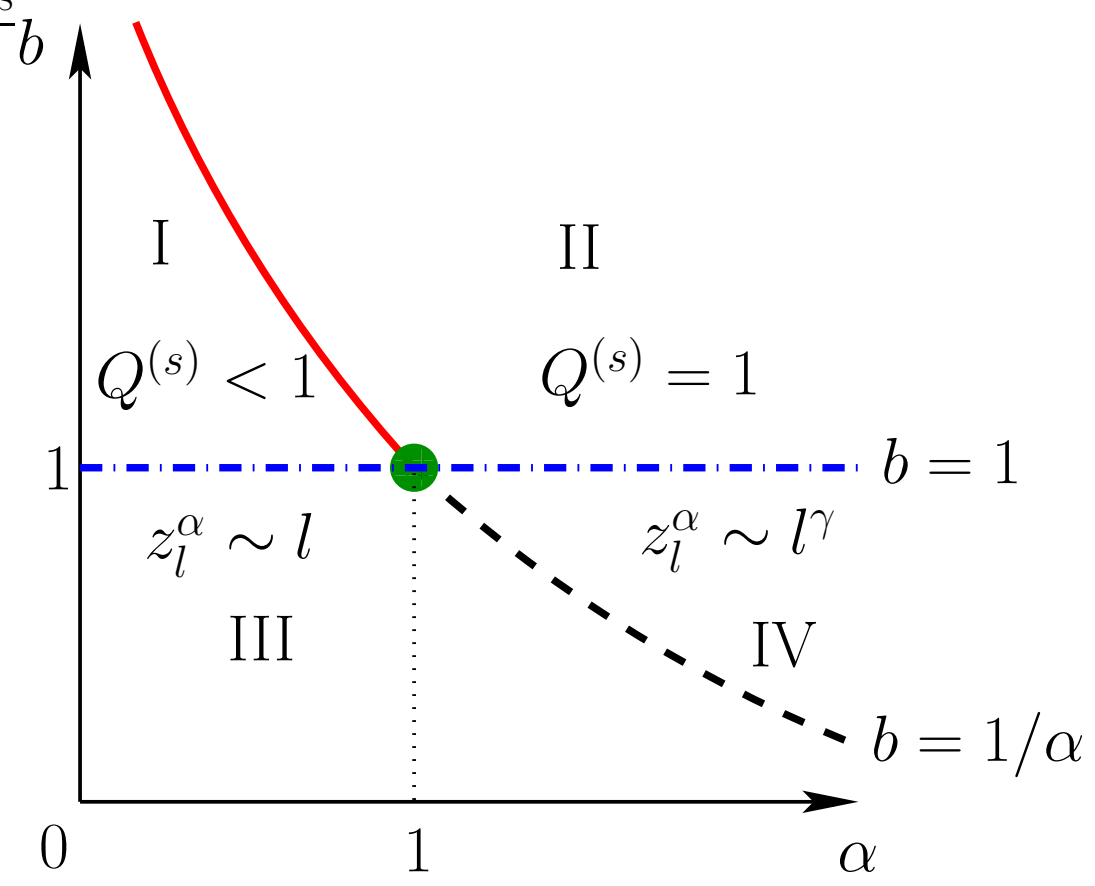

Figure 10. Phase diagram summarizing our results for Gumbel class distributions. Four different regions I, II, III, and IV are identified, depending on the behavior of $\tilde{z}_{l}$ and $Q^{(s)}$ in (74).

$Q^{(s)}=0$ and $z_{l}^{\alpha}=\tilde{z}_{l}^{\alpha}=l-o(l)$, whereas $Q^{(s)}=1$ for $\alpha>1$. At $\alpha=b=1$ (represented as a green filled circle in figure 10), $Q^{(s)}$ changes its behavior discontinuously from 0 $(c \leq a)$ to $1(c>a)$ similar to the behavior along the line $b=1 / \alpha$ with $b>1$. Unlike the case of $b>1$, however, $\rho(x)$ is not equal to the density of the background process when $Q^{(s)}=1$. Moreover, at the critical point $c=a$, the mean record value increases anomalously slowly as $z_{l} \sim \sqrt{l}$. For further details we refer the reader to [14].

In both regions III and IV $(b<1), Q^{(s)}=0$ and $z_{l}=\tilde{z}_{l}$ diverges. The difference between region III and IV is characterized by the asymptotic behavior of $z_{l}^{\alpha}$. In region III $z_{l}^{\alpha}=l-o(l)$ like in the standard record process, while in region IV $z_{l}^{\alpha} \sim l^{\gamma}$ with $\gamma=(1-b) /(1-1 / \alpha)<1$. On the borderline between regions III and IV, which is represented by a black dashed line in figure 10, $z_{l}^{\alpha}=A l-o(l)$ with a $c$-dependent constant $A$ which is the positive solution of (16) and decreases continuously from $A=1$ at $c=0$ to $A=0$ for $c \rightarrow \infty$. The point $\alpha=b=1$ is again special in that here the amplitude $A$ vanishes at a finite value $c=a$ [14]. When interpreted in terms of the amplitude $A$ of the diverging component in (74), the transition to the stationary phase is continuous at $\alpha=b=1$ but discontinuous for $1 / \alpha=b>1$.

The behavior for distributions in the Fréchet and Weibull classes corresponds roughly to that of Gumbel-class distributions with very heavy $(\alpha \rightarrow 0)$ and light $(\alpha \rightarrow \infty)$ tails, respectively. Specifically, for the Fréchet class distributions the handicap is irrelevant when $b<1$. For $b>1$ the stochastic bistability scenario observed for the Gumbel class applies and $Q^{(s)}$ is generally nonzero. An exception are heavy tailed 
distributions with tail exponent $\mu<1 /(b-1)$, where the fluctuations of the background process are sufficiently strong to overcome the increasing handicap and $Q^{(s)}=0$. This is reminiscent of the problem of records from i.i.d. sequences with a linear trend, where the record process becomes asymptotically stationary only if the underlying distribution has a finite first moment [11. For distributions with bounded support belonging to the Weibull class, all but a finite number of random variables become records for $b>1$. When $b<1$, the approach of the mean record value to the boundary $r$ of the support is dominated by the handicap in the sense that $r-z_{l} \sim \delta_{l}$, and the tail behavior of the density of record values is found to be the same as that of the density $f_{w}$ of the background process.

An important motivation for our study comes from the connection to adaptive walks in rugged fitness landscapes with deterministic epistasis in the sense of [28]. In section 5 ] the results obtained for the $\delta$-exceedance records were used to quantify the increase in the mean walk length that is caused by the increasing deterministic fitness profile $g_{k}$ in (2). For $b>1$ the stochastic bistability of the record process implies that the distribution of walk lengths becomes bimodal. With a finite probability $Q^{(s)}$, walks traverse the entire fitness landscape and reach the maximal possible length $O(L)$. In the case $b<1$ which corresponds to the biologically important scenario of diminishing returns epistasis, the effect on the walk length is more subtle and depends sensitively on the distribution of the random fitness component. In the Fréchet class and in the Gumbel class with $\alpha<1 / b$ the asymptotic behavior is $D_{\mathrm{RAW}} \sim \ln L$ as on an uncorrelated landscape, whereas for light-tailed Gumbel class distributions with $\alpha>1 / b$ there is a slight increase in the walk length which now grows as $(\ln L)^{1 / \gamma}$ with $\gamma<1$. By contrast, the walk length for the Weibull class grows at least as a power law in $L$ and is given by

$$
D_{\mathrm{RAW}} \sim \begin{cases}L & \text { if } \nu>1-b \\ (L / \ln L)^{\nu /(1-b)} & \text { if } \nu \leq 1-b .\end{cases}
$$

We note that these results are potentially relevant for the interpretation of microbial evolution experiments, where examples of fitness distributions belonging to each of the three EVT classes have been identified empirically [30, 31, 32, 33].

The analysis of adaptive walk length also provides some insight into the statistics of record occurrence times in the $\delta$-exceedance record process, which we have not explicitly addressed in this work. As was explained in section 1.2, the walk length $D_{\mathrm{RAW}}(L)$ is expected to be of the same order as the number of record events up to time $n=L$, a relation that can be made precise for a particular variant of the adaptive walk problem called 'simple' adaptive walk in [18]. This relation reproduces the fact that a finite fraction of random variables are records when $D_{\mathrm{RAW}}(L) \sim L$, and can be used to estimate the rate of record occurrence in the other cases analyzed in this paper. A detailed analysis of the temporal statistics of the $\delta$-exceedance process, including in particular the question of correlations between record events [34, 35], appears to be an interesting problem for future study. 


\section{Acknowledgments}

We thank Satya Majumdar for useful discussions. S-CP acknowledges the support by the Basic Science Research Program through the National Research Foundation of Korea (NRF) funded by the Ministry of Science, ICT and Future Planning (Grant No. 2014R1A1A2058694); and by The Catholic University of Korea, Research Fund, 2016. JK acknowledges the support of DFG within SPP 1590, and the kind hospitality of the University of Florence during the completion of this work.

\section{Appendix A. Derivation of (8)}

In this appendix, we derive the recursion relations for the mean $z_{l}$ and the variance $V_{l}$ of $Q_{l}(x)$ from (7). We consider a bounded density $f(x)$ with the support $0<x<R$, and the case with unbounded support will be obtained by taking the limit $R \rightarrow \infty$.

We first introduce the moment generating function

$$
G_{l}(\lambda)=\int_{0}^{R} e^{-\lambda x} Q_{l}(x) d x
$$

which gives

$$
z_{l}=-\left.\frac{d G_{l}}{d \lambda}\right|_{\lambda=0}, \quad V_{l}=\left.\frac{d^{2} \ln G_{l}}{d \lambda^{2}}\right|_{\lambda=0} .
$$

For convenience, we introduce

$$
F_{c}(x)=1-F(x), \quad \tilde{F}(x)=e^{-\lambda x} F_{c}(x),
$$

and we define $F(x)=0$ if $x<0$. Later, we will also use the following identity,

$$
e^{-\lambda x} f(x)=-\frac{d \tilde{F}(x)}{d x}-\lambda \tilde{F}(x) .
$$

Note that $Q_{l+1}(y)$ for $y>R-\delta_{l}$ is given by

$$
Q_{l+1}(y)=I_{0} f(y)
$$

where

$$
I_{0} \equiv \int_{0}^{R} \frac{Q_{l}(x)}{1-F\left(x-\delta_{l}\right)} d x
$$

which does not depend on $y$.

We now insert (A.1) into the recursion (7). After separating the integration domain followed by integration by parts, $G_{l+1}(\lambda)$ can be written as

$$
\begin{aligned}
G_{l+1}(\lambda) & =\int_{0}^{R-\delta_{l}} e^{-\lambda y} Q_{l+1}(y) d y+\int_{R-\delta_{l}}^{R} e^{-\lambda y} Q_{l+1}(y) d y \\
& =\int_{0}^{R-\delta_{l}} d y e^{-\lambda y} f(y) \int_{0}^{y+\delta_{l}} \frac{Q_{l}(x)}{F_{c}\left(x-\delta_{l}\right)}+I_{0} \int_{R-\delta_{l}}^{R} d y e^{-\lambda y} f(y) \\
& =I_{1}+I_{0} \tilde{F}\left(R-\delta_{l}\right)-\lambda I_{0} \int_{R-\delta_{l}}^{R} d y \tilde{F}(y),
\end{aligned}
$$


with

$$
\begin{aligned}
I_{1} \equiv & \int_{0}^{R-\delta_{l}} d y e^{-\lambda y} f(y) \int_{0}^{y+\delta_{l}} \frac{Q_{l}(x)}{F_{c}\left(x-\delta_{l}\right)} d x \\
= & \int_{0}^{R-\delta_{l}} e^{-\lambda y} f(y) d y \int_{0}^{\delta_{l}} Q_{l}(x) d x+\int_{0}^{R-\delta_{l}} d y e^{-\lambda y} f(y) \int_{\delta_{l}}^{y+\delta_{l}} \frac{Q_{l}(x)}{F_{c}\left(x-\delta_{l}\right)} d x \\
= & {\left[1-\tilde{F}\left(R-\delta_{l}\right)-\lambda \int_{0}^{R-\delta_{l}} d y \tilde{F}(y)\right] \int_{0}^{\delta_{l}} Q_{l}(x) d x } \\
& +\int_{0}^{R-\delta_{l}} d y e^{-\lambda y} f(y) \int_{0}^{y} \frac{Q_{l}\left(x+\delta_{l}\right)}{F_{c}(x)} d x,
\end{aligned}
$$

where we have changed the variable $x \mapsto x+\delta_{l}$. Changing the order of integration and then integrating by parts, the last integral in (A.8), to be denoted by $I_{2}$, becomes

$$
\begin{aligned}
I_{2}= & \int_{0}^{R-\delta_{l}} d x \frac{Q_{l}\left(x+\delta_{l}\right)}{F_{c}(x)} \int_{x}^{R-\delta_{l}} d y e^{-\lambda y} f(y) \\
= & \int_{\delta_{l}}^{R} e^{-\lambda\left(x-\delta_{l}\right)} Q_{l}(x) d x-\tilde{F}\left(R-\delta_{l}\right) \int_{\delta_{l}}^{R} d x \frac{Q_{l}(x)}{F_{c}\left(x-\delta_{l}\right)} \\
& -\lambda \int_{0}^{R-\delta_{l}} d y \tilde{F}(y) \int_{\delta_{l}}^{y+\delta_{l}} \frac{Q_{l}(x)}{F_{c}\left(x-\delta_{l}\right)} d x .
\end{aligned}
$$

The last integral in (A.9), to be denoted by $I_{3}$, can be written as

$$
I_{3}=-\lambda \int_{0}^{R-\delta_{l}} e^{-\lambda y} \frac{Q_{l+1}(y)}{h(y)} d y+\lambda \int_{0}^{R-\delta_{l}} d y \tilde{F}(y) \int_{0}^{\delta_{l}} Q_{l}(x) d x,
$$

where $h(x)=f(x) / F_{c}(x)$ is the hazard function. Hence we get

$$
\begin{aligned}
G_{l+1}(\lambda) & =\int_{\delta_{l}}^{R} e^{-\lambda\left(x-\delta_{l}\right)} Q_{l}(x) d x+\int_{0}^{\delta_{l}} Q_{l}(x) d x-\lambda \int_{0}^{R} e^{-\lambda x} \frac{Q_{l+1}(x)}{h(x)} d x \\
& =e^{\lambda \delta_{l}} G_{l}(\lambda)-\lambda \int_{0}^{R} e^{-\lambda x} \frac{Q_{l+1}(x)}{h(x)} d x+\int_{0}^{\delta_{l}}\left(1-e^{-\lambda\left(x-\delta_{l}\right)}\right) Q_{l}(x) d x,
\end{aligned}
$$

where we have used (A.5) for $y>R-\delta_{l}$. Note that even if $R=\infty$ the above relation is still valid.

For the first moment, if it exists, we get

$$
\begin{aligned}
z_{l+1} & =-\left.\frac{d G_{l+1}}{d y}\right|_{\lambda=0}=z_{l}-\delta_{l}+\int_{0}^{R} \frac{Q_{l+1}(x)}{h(x)} d x-\int_{0}^{\delta_{l}}\left(x-\delta_{l}\right) Q_{l}(x) d x \\
& =\int_{0}^{R} \frac{Q_{l+1}(x)}{h(x)} d x+\int_{\delta_{l}}^{R}\left(x-\delta_{l}\right) Q_{l}(x) d x
\end{aligned}
$$

and for the second moment $\xi_{l}$, if it exists, we get

$$
\begin{aligned}
\xi_{l+1} & =\left.\frac{d^{2} G_{l+1}}{d y^{2}}\right|_{\lambda=0} \\
& =\xi_{l}-2 \delta_{l} z_{l}+\delta_{l}^{2}+2 \int_{0}^{R} x \frac{Q_{l+1}(x)}{h(x)} d x-\int_{0}^{\delta_{l}}\left(x-\delta_{l}\right)^{2} Q_{l}(x) d x
\end{aligned}
$$

which gives the recursion relation for the second cumulant, or variance, $V_{l}=\xi_{l}-z_{l}^{2}$ as $V_{l+1}-V_{l}=\left(z_{l}-\delta_{l}\right)^{2}-z_{l+1}^{2}+2 \int_{0}^{R} x \frac{Q_{l+1}(x)}{h(x)} d x-\int_{0}^{\delta_{l}}\left(x-\delta_{l}\right)^{2} Q_{l}(x) d x$. 
If the support is unbounded on both sides, we get

$$
\begin{aligned}
G_{l+1}(\lambda) & =\int_{-\infty}^{\infty} e^{-\lambda y} Q_{l+1}(y) d y=\int_{-\infty}^{\infty} d x \frac{Q_{l}(x)}{F_{c}\left(x-\delta_{l}\right)} \int_{x-\delta_{l}}^{\infty} e^{-\lambda y} f(y) d y \\
& =e^{\lambda \delta_{l}} G_{l}(\lambda)-\lambda \int_{-\infty}^{\infty} d x \frac{Q_{l}(x)}{F_{c}\left(x-\delta_{l}\right)} \int_{x-\delta_{l}}^{\infty} \tilde{F}(y) d y \\
& =e^{\lambda \delta_{l}} G_{l}(\lambda)-\lambda \int_{-\infty}^{\infty} e^{-\lambda x} \frac{Q_{l+1}(x)}{h(x)} d x
\end{aligned}
$$

which is (A.11) without the last integral.

\section{Appendix B. Analysis of the variance for decreasing handicaps in the Gumbel class}

In this appendix, we will show that the MFA used in section 3 is valid in the sense that $V_{l} / z_{l}^{2} \rightarrow 0$ as $l \rightarrow \infty$. Since the variance $V_{l}$ is not neglected, we relax the MFA in such a manner that $Q_{l}(x)=q\left[\left(x-z_{l}\right) / \sigma_{l}\right] / \sigma_{l}$, where $\sigma_{l} \equiv \sqrt{V_{l}}$ is the standard deviation and $q(y)$ is supposed to be independent of $l$. Note that the mean and variance of $q(y)$ are 0 and 1 , respectively. We are still assuming that $Q_{l}(x)$ has a well-defined steady state distribution when $x$ is appropriately rescaled. Recalling that the hazard function for the Gumbel class distributions is $h(x)=\alpha x^{\alpha-1} / a$, see (10), we may thus approximate the integral terms on the right hand sides of (A.12) and (A.14) as

$$
\begin{aligned}
\int d x x^{n-\alpha} Q_{l+1}(x) & =\int d t\left(z_{l+1}+\sigma_{l+1} t\right)^{n-\alpha} q(t) \\
& \approx z_{l+1}^{n-\alpha}\left[1+\frac{(n-\alpha)(n-1-\alpha)}{2} \frac{V_{l+1}}{z_{l+1}^{2}}\right],
\end{aligned}
$$

where we have assumed $\sigma_{l} / z_{l} \ll 1$. Note that (B.1) is exact when $n=\alpha$ or $n=\alpha+1$.

From (A.12) and (A.14) along with (B.1), we get

$$
\begin{aligned}
& z_{l+1}-z_{l}=\frac{a}{\alpha} z_{l+1}^{1-\alpha}\left[1+\frac{\alpha(\alpha-1)}{2} \frac{V_{l+1}}{z_{l+1}^{2}}\right]-\delta_{l}, \\
& V_{l+1}-V_{l}=\left(z_{l}-\delta_{l}\right)^{2}-z_{l+1}^{2}+\frac{2 a}{\alpha} z_{l+1}^{2-\alpha}\left[1+\frac{(2-\alpha)(1-\alpha)}{2} \frac{V_{l+1}}{z_{l+1}^{2}}\right] .
\end{aligned}
$$

Using the result (20) of the MFA, we see that $V_{l}$ contributes at best to the subleading behavior in (B.2) if $V_{l} \approx\left(C l^{\epsilon}\right)^{2}$ with $\epsilon<\gamma / \alpha$. The leading behavior of $V_{l}$, or the value of $\epsilon$, will be determined from $(\underline{B .3})$.

Before finding $\epsilon$, we rewrite $(\underline{B .3})$ using $(\underline{B .2})$ as

$$
\begin{aligned}
V_{l+1}-V_{l} & \approx\left(z_{l}-\delta_{l}\right)^{2}-z_{l+1}^{2}+2 z_{l+1}\left(z_{l+1}-z_{l}+\delta_{l}\right)\left[1+(1-\alpha) \frac{V_{l+1}}{z_{l+1}^{2}}\right] \\
& =\left(z_{l+1}-z_{l}+\delta_{l}\right)^{2}+2(1-\alpha)\left(z_{l+1}-z_{l}+\delta_{l}\right) \frac{V_{l+1}}{z_{l+1}} \\
& =\frac{1}{h\left(z_{l+1}\right)^{2}}-2(\alpha-1) \frac{V_{l+1}}{z_{l+1} h\left(z_{l+1}\right)}
\end{aligned}
$$

where we have used (11) and only kept the leading terms. 


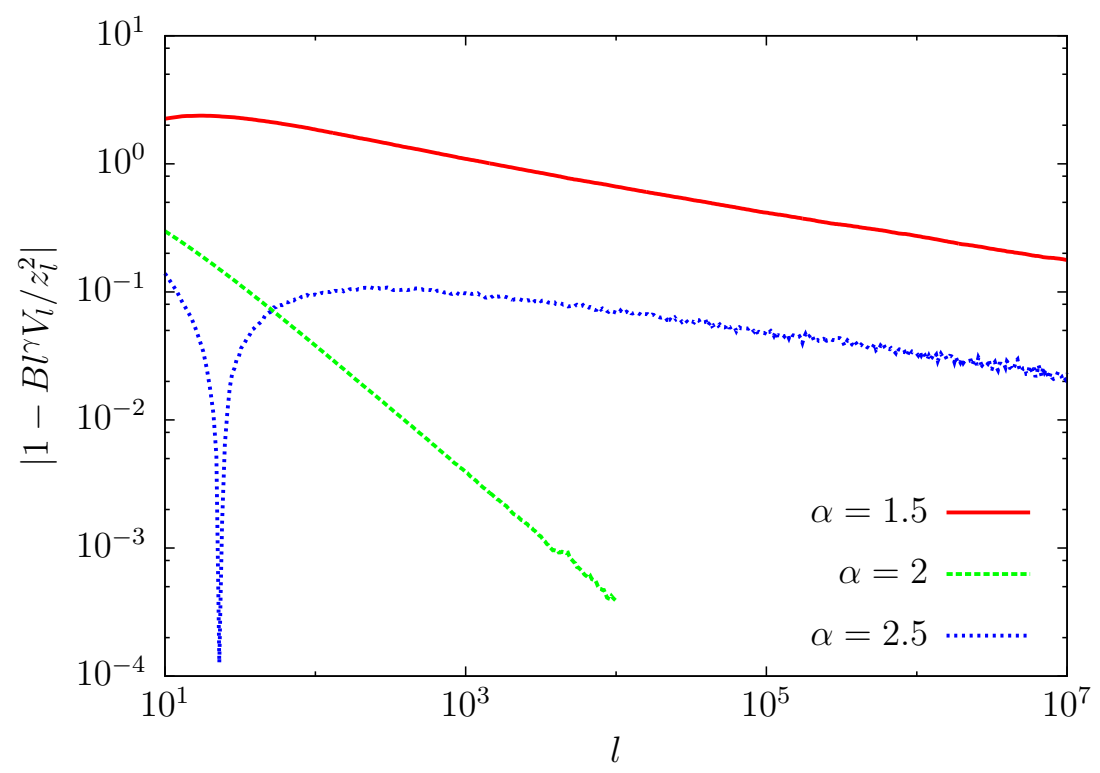

Figure B1. Double logarithmic plots of $\left|1-B l^{\gamma} V_{l} / z_{l}^{2}\right|$ vs $l$ for $\alpha=1.5,2,2.5$ with $b=\frac{1}{2}, a=c=1$. All curves decay to zero with different powers.

For $\alpha \leq 1 / b$ we have $h\left(z_{l}\right)=\alpha A l / z_{l}=\alpha(A l)^{1-1 / \alpha} / a^{1 / \alpha}$, and assuming $V_{l} \approx C^{2} l^{2 \epsilon}$ we obtain

$$
2 \epsilon C^{2} l^{2 \epsilon-1} \doteq \frac{a^{2 / \alpha}}{\alpha^{2}}(A l)^{-2+2 / \alpha}-2 C^{2} \frac{\alpha-1}{\alpha A} l^{2 \epsilon-1},
$$

which gives

$$
\epsilon=\frac{1}{\alpha}-\frac{1}{2}, \quad C=\frac{(A a)^{1 / \alpha}}{\alpha A \sqrt{1+2\left(A^{-1}-1\right)(1-1 / \alpha)}} .
$$

Since $A=1$ if $\alpha<1 / b$ and $A<1$ if $1 / \alpha=b<1, C$ is positive.

Next we consider the case $\alpha>1 / b$, where $h\left(z_{l}\right)^{-1}=z_{l}(A l)^{-\gamma} / \alpha=$ $a^{1 / \alpha}(A l)^{(1 / \alpha-1) \gamma} / \alpha$, and hence

$$
2 \epsilon C^{2} l^{2 \epsilon-1} \doteq \frac{a^{2 / \alpha}(A l)^{2(1 / \alpha-1) \gamma}}{\alpha^{2}}-2 C^{2} \frac{\alpha-1}{\alpha A^{\gamma}} l^{2 \epsilon-\gamma} .
$$

Since $\gamma<1$, the left hand side can at most contribute to the subleading behavior. Thus, we get

$$
\epsilon=\frac{\gamma}{\alpha}-\frac{\gamma}{2}, \quad C=\left[\frac{\left(a A^{\gamma}\right)^{2 / \alpha}}{2 \alpha(\alpha-1) A^{\gamma}}\right]^{1 / 2} .
$$

To sum up, we found that

$$
\frac{V_{l}}{z_{l}^{2}} \approx \frac{l^{-\gamma}}{B}
$$

with

$$
B= \begin{cases}\alpha^{2}\left[A^{2}+2 A(1-A)(1-1 / \alpha)\right], & \alpha \leq 1 / b \\ 2 \alpha(\alpha-1) A^{\gamma}, & \alpha>1 / b\end{cases}
$$


where $\gamma$ and $A$ are given in (22) and (21). Hence, the MFA becomes exact as $l \rightarrow \infty$.

To confirm the above prediction, we performed Monte Carlo simulations for various

values of $b, \alpha$, and $c$. In figure B1, we depict the deviation $\left|1-B V_{l} l^{\gamma} / z_{l}^{2}\right|$ from (B.9)

against $l$ for $b=\frac{1}{2}, c=1$, and $\alpha=1.5,2,2.5$ on a double-logarithmic scale. As predicted, all curves approach zero.

\section{References}

[1] Glick N 1978 Amer. Math. Monthly 85 2-26

[2] Arnold B, Balakrishnan N and Nagaraja H 1998 Records (New York: Wiley)

[3] Wergen G 2013 J. Phys. A: Math. Theor. 46223001

[4] Edery Y, Kostinski A B, Majumdar S N and Berkowitz B 2013 Phys. Rev. Lett. 110180602

[5] Gouet R, López F J and Sanz G 2007 Bernoulli 13 754-781

[6] Gouet R, López F J and Sanz G 2012 Test 21 188-214

[7] Balakrishnan N, Pakes A and Stepanov A 2005 Adv. Appl. Probab. 37 765-780

[8] Vervaat J 1973 Stochastic Process. Appl. 1 317-334

[9] Gouet R, López F J and Sanz G 2005 Adv. Appl. Probab. 37 781-800

[10] Wergen G, Volovik D, Redner S and Krug J 2012 Phys. Rev. Lett. 109164102

[11] Ballerini R and Resnick S I 1987 Adv. Appl. Probab. 19801

[12] Franke J, Wergen G and Krug J 2010 J. Stat. Mech.:Theory Exp. P10013

[13] Balakrishnan N, Balasubramanian K and Panchapakesan S 1996 J. Appl. Stat. Sci. 4 123-132

[14] Park S C, Szendro I G, Neidhart J and Krug J 2015 Phys. Rev. E 91042707

[15] Kauffman S and Levin S 1987 J. Theor. Biol. 128 11-45

[16] Macken C A and Perelson A S 1989 Proc. Nat. Acad. Sci. USA 86 6191-6195

[17] Flyvbjerg H and Lautrup B 1992 Phys. Rev. A 46 6714-6723

[18] Orr H A 2002 Evolution 56 1317-1330

[19] Neidhart J and Krug J 2011 Phys. Rev. Lett. 107178102

[20] Seetharaman S and Jain K 2014 Phys. Rev. E 90032703

[21] Neidhart J, Szendro I G and Krug J 2014 Genetics 198 699-721

[22] de Visser J A G M, Cooper T F and Elena S F 2011 Proc. R. Soc. Lond. Ser. B 278 3617-3624

[23] Chou H H, Chiu H C, Delaney N F, Segrè D and Marx C J 2011 Science 332 1190-1192

[24] Khan A I, Dinh D M, Schneider D, Lenski R E and Cooper T F 2011 Science 332 1193-1196

[25] Wiser M J, Ribeck N and Lenski R E 2013 Science 342 1364-1367

[26] Berger D and Postma E 2014 Genetics 198 1417-1420

[27] Couce A and Tenaillon O 2015 Frontiers in Genetics 699

[28] Wiehe T 1997 Genet. Res. Camb. 69 127-136

[29] de Haan L and Ferreira A 2006 Extreme Value Theory (Berlin: Springer)

[30] Kassen R and Bataillon T 2006 Nat. Gen. 38 484-488

[31] Rokyta D, Beisel C, Joyce P, Ferris M, Burch C and Wichman H 2008 J. Mol. Evol. 67 368-376

[32] Schenk M, Szendro I, Krug J and de Visser J 2012 PLoS Genet. 8 e1002783

[33] Bank C, Hietpas R, Wong A, Bolon D and Jensen J 2014 Genetics 196 841-852

[34] Wergen G, Franke J and Krug J 2011 J. Stat. Phys. 144 1206-1222

[35] Franke J, Wergen G and Krug J 2012 Phys. Rev. Lett. 108064101 